KMU FORSCHUNG AUSTRIA

Austrian Institute for SME Research

\title{
Zweite begleitende Evaluierung des „ImpulsProgramm creativwirtschaft“ der aws
}




\section{KMU FORSCHUNG AUSTRIA}

Austrian Institute for SME Research

Diese Studie wurde im Auftrag der Austria Wirtschaftsservice $\mathrm{GmbH}$ durchgeführt.

\section{Verfasser/innen des Berichts: \\ Alfred Radauer (Projektleiter) \\ Aliette Dörflinger \\ Internes Review/Begutachtung: \\ Sonja Sheikh}

Layout:

Martina Gugerell

Die vorliegende Studie wurde nach allen Maßstäben der Sorgfalt erstellt.

Die KMU FORSCHUNG AUSTRIA übernimmt jedoch keine Haftung für Schäden oder Folgeschäden, die auf diese Studie oder auf mögliche fehlerhafte Angaben zurückgehen.

Dieses Werk ist urheberrechtlich geschützt. Jede Art von Nachdruck, Vervielfältigung, Verbreitung, Wiedergabe, Übersetzung oder Einspeicherung und Verwendung in Datenverarbeitungssystemen, und sei es auch nur auszugsweise, ist nur mit ausdrücklicher Zustimmung der KMU FORSCHUNG AUSTRIA gestattet. 


\section{Inhaltsverzeichnis}

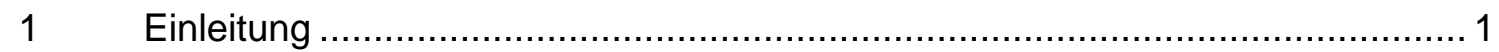

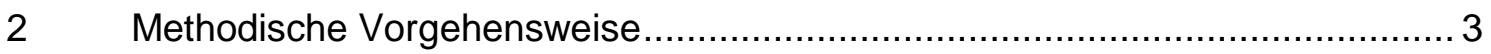

3 Beschreibung des Evaluierungsgegenstandes ........................................ 5

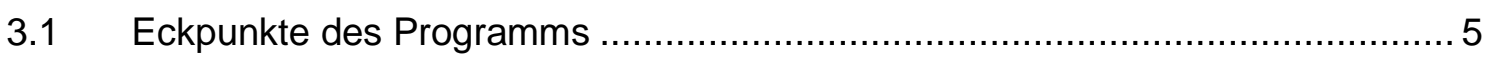

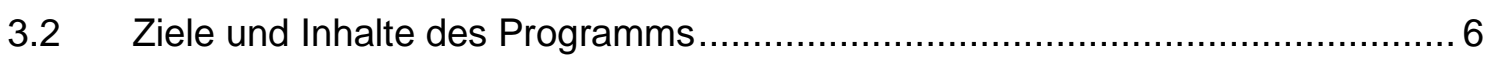

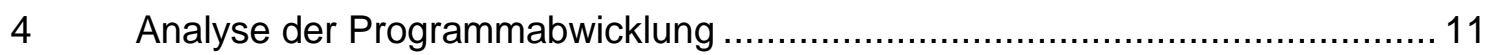

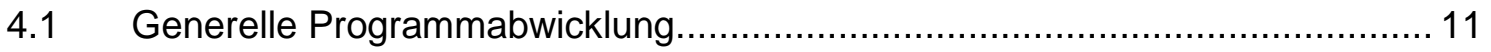

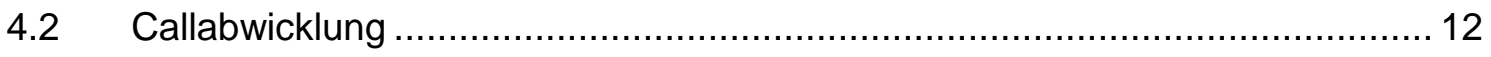

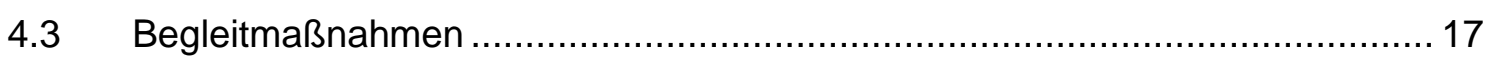

$5 \quad$ Analyse der Mobilisierungswirkungen des Programms................................ 24

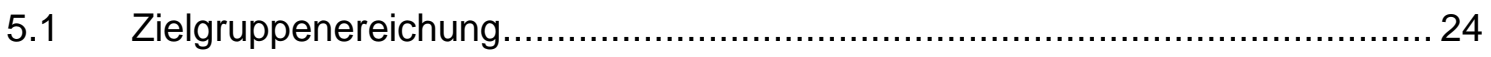

$5.2 \quad$ Struktur der bei iP teilnehmenden Unternehmen ........................................ 28

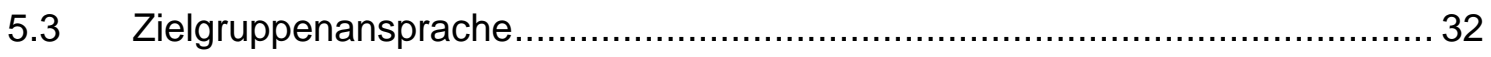

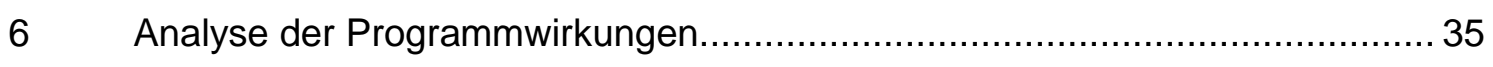

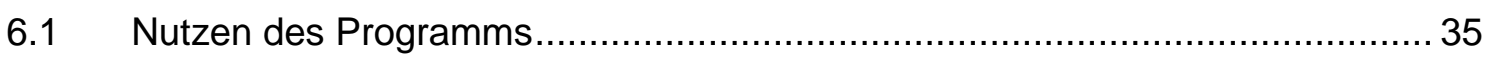

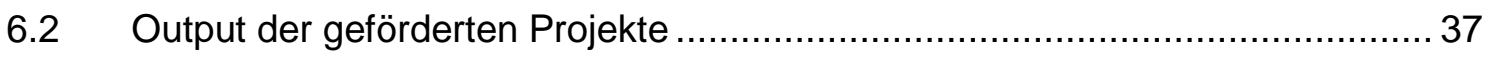

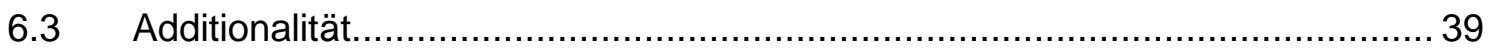

$7 \quad$ Zusammenfassung und Schlussfolgerungen ........................................... 40

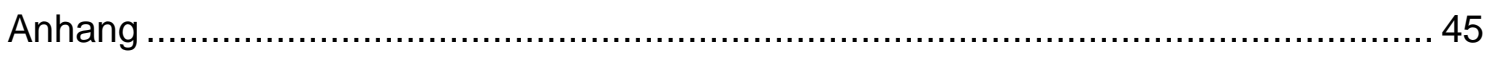

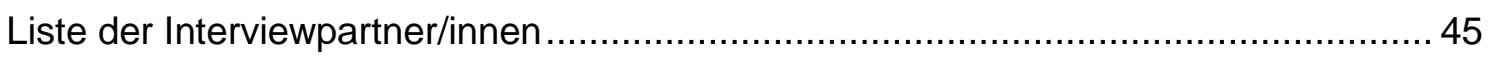




\section{Grafikverzeichnis}

Grafik 1

Grafik 2

Grafik 3

Grafik 5

Grafik 6

Grafik 7

Grafik 8

Grafik 9

Grafik 10

Grafik 11

Grafik 12

Grafik 13

Grafik 14

Grafik 15

Grafik 16

Grafik 17

Grafik 18

Grafik 19

Logic Chart für das ImpulsProgramm creativwirtschaft 7

Zufriedenheit mit Aspekten der generellen Programmabwicklung, auf einer Skala von $1=$ sehr zufrieden bis $4=$ gar nicht zufrieden, arithmetische Mittelwerte der Antworten der einreichenden Betriebe aus dem 2. und 3. Call

Zufriedenheit mit Aspekten der Antragstellungsphase, auf einer Skala von $1=$ sehr zufrieden bis $4=$ gar nicht zufrieden, arithmetische Mittelwerte der Antworten der einreichenden Betriebe aus dem 2. und 3. Call

Zufriedenheit mit Aspekten der Förderabwicklung, auf einer Skala von $1=$ sehr zufrieden bis 4= gar nicht zufrieden, arithmetische Mittelwerte der Antworten der geförderten Betriebe aus dem 1. und 2. Call......... 15 Inanspruchnahme und Zufriedenheit mit den iP Workshops, Betriebe in

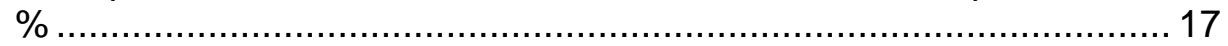

Gründe der Nichtteilnahme an den Begleitmaßnahmen von iP, Betriebe in \%, Mehrfachnennungen möglich.................................... 19

Gründe der Nicht-Teilnahme am we-Workshop, Betriebe in \% .......... 20

Bereiche mit Weiterbildungsbedarf, Betriebe in \% ............................ 23

Anzahl der Einreichungen, nach Call und Bereich ........................... 25

Regionale Verteilung der Einreichungen, nach Bundesländer und nach Call, in \% .......................................................................... 27

Anzahl der Mitarbeiter/innen bei den Unternehmen des 1. Calls (Förderempfänger), 2. und 3. Call (Förderempfänger und Einreicher), Betriebe in \% .....

Umsatz im Jahr 2006 der einreichenden Unternehmen des 1., 2. und 3. Calls, Betriebe in \%

Formen der Beschäftigung bei den einreichenden Unternehmen des 1 ., 2. und 3. Calls, Betriebe in \%, Mehrfachnennungen möglich .

Unternehmenstätigkeit der einreichenden Unternehmen des 2. und 3.

Calls, Betriebe in \% 30

Teilnahme an Förderprogrammen und/oder Beziehen von Preisen/ Stipendien in den letzten 5 Jahren, Betriebe in \%.....

Einschätzung der Bekanntheit von iP durch einreichende Betriebe, Betriebe in \% ....

Bekanntheitsgrad der Leitprojekte und deren Bezug zu iP, einreichende Betriebe des 1., 2. und 3. Calls in \%.

Informationskanäle, über die die Einreicher des 2. und 3. Calls von iP erfahren haben, Betriebe in \%

Nutzendimensionen der iP Förderung bei den geförderten

Unternehmen des 1. und 2. Call, Betriebe in \%. 
Grafik 20 Resultate der Entwicklungstätigkeiten der geförderten Unternehmen des 1. und 2. Call, Betriebe in \%

Grafik $21 \quad$ Nutzung von IPR in den geförderten Projekten des 1. und 2. Call,

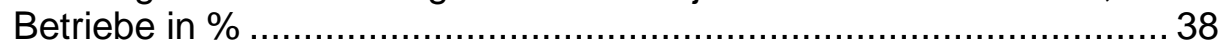

Grafik 22 Additionalität, Anzahl der geförderten Unternehmen absolut. 39

\section{Tabellenverzeichnis}

Tabelle 1 Rücklaufquoten der Online-Befragung der an iP teilnehmenden Unternehmen, nach Call und nach Förderempfänger/Einreicher. 3

Tabelle 2 Anzahl und Projektkosten der geförderten und eingereichten Projekte 25

Tabelle 3 Anzahl begründeter Schutzrechte bei geförderten Projekten des 1. und 2. Call, Absolutzahlen 38 


\section{$1 \quad$ Einleitung}

Zahlreiche internationale Studien weisen darauf hin, dass die Kreativwirtschaft in vielen Ländern einen erheblichen Wirtschaftsfaktor darstellt. Dies gilt auch für Österreich: So wurde im Zweiten Österreichischen Kreativwirtschaftsbericht festgehalten, dass die „creative industries“ (Cl) im Jahr 2004 etwa 30.000 Betriebe umfasste, insgesamt rd. 100.000 Personen beschäftigte und Erlöse und Erträge von etwa $€ 18,3$ Mrd. erwirtschaftete. Die erfolgreiche Markteinführung von Innovationen stellt für das Wachstum der Kreativwirtschaft hierbei eine wichtige Erfolgsgröße dar. Allerdings steht in den $\mathrm{Cl}$ einer erfolgreichen Innovationstätigkeit vielfach eine gewisse Reserviertheit gegenüber Technik und wirtschaftlich orientierten Unternehmertum (bedingt durch eine stärkere Betonung des Kunstaspektes) sowie die kleinstbetriebliche Struktur mit ihren Ressourcenbeschränkungen gegenüber.

Vor diesem Hintergrund wurde im Jahr 2004 das ImpulsProgramm creativwirtschaft (iP) ins Leben gerufen. Ziel des Programms ist es, die Wettbewerbsfähigkeit und das Innovationspotenzial von KMU, die in der Kreativwirtschaft tätig sind, zu stärken. Hierfür wurde in der Anfangsphase primär auf einen projektbezogenen monetären Zuschuss, der im Rahmen von Calls - flankiert durch Qualifizierungsmaßnahmen - vergeben wurde als Förderinstrument zurückgegriffen. Das diesbezüglich zur Verfügung stehende Budget lag für die Jahre 2004 und 2005 bei rd. € 3,8 Mio. Im Zeitraum Juni 2005 bis Jänner 2006 wurde iP einer ersten begleitenden Evaluierung unterzogen, die den 1. Call abdeckte. Diese Evaluierung stellte dem Programm ein insgesamt gutes Zeugnis aus und formulierte einige Ansatzpunkte für Verbesserungen (z.B. hinsichtlich des Spannungsfeldes künstlerischer Anspruch und technischer Innovationsgehalt oder bezüglich eines differenzierten Vorgehens in den drei Förderbereichen Design, Musik und Multimedia, auf Grund der stark differierenden Qualität der Anträge in den genannten Bereichen).

Seit Januar 2006 wurde iP deutlich weiterentwickelt. Der monetäre Zuschuss (nunmehr „iP Förderung“ genannt) bildet zwar weiterhin die zentrale Säule des Programms (bislang wurden drei Calls durchgeführt), doch wurde das Leistungsspektrum insbesondere im Bereich der Qualifizierungsmaßnahmen - mit der Abhaltung der Workshopreihe „we - workshops for entrepreneurs" und der iP Workshopreihe „Businessplan“ und „Einreichberatung“ mit Cl-spezifischen Inhalten - erweitert. Gleichzeitig sollen durch neu konzipierte "Leitprojekte“ gemeinsam mit Kooperationspartnern weiterführende Maßnahmen zur Stärkung und Vermarktung des $\mathrm{Cl}$ Standortes Österreich implementiert werden. Bislang wurden vier derartige Leitprojekte initiiert: das „Austrian Music Ambassador Network (AMAN)“, das „designforum“, „Kreatives Handwerk Tirol“ und "Kreatives Handwerk Salzburg“. Auf organisatorischer Ebene erfolgte u. a. eine vollständige Eingliederung des Programms in das Leistungsportfolio der aws, (Abschluss Herbst 2007) - die Wirtschaftskammer Österreich fungiert nur noch als Programmpartner. Die Büros des Programmmanagements wurden vom Museumsquartier im 7. Wiener Gemeindebezirk (das eine Nähe zur Zielgruppe der Künstler ermöglich hat) in die Räumlichkeiten der aws verlegt, um Synergieeffekte mit anderen Abteilungen der aws zu ermöglichen. 
Vor dem Hintergrund der oben genannten Änderungen und der Weiterentwicklungen hat die Austria Wirtschaftsservice $\mathrm{GmbH}$ (aws) die KMU FORSCHUNG AUSTRIA beauftragt, das Programm einer neuerlichen Überprüfung zu unterziehen. Im Rahmen der Evaluierung war insbesondere zu klären, ob das Förderprogramm hinsichtlich der Erreichung seines Globalzieles - der Steigerung des Innovationspotenzials und der Wettbewerbsfähigkeit von österreichischen KMU in und durch die Bereiche Musik, Multimedia und Design - in seiner Implementierung als effektiv und effizient angesehen werden kann. Auf Basis dieser Analysen galt es, etwaige Schwachstellen des Programms zu identifizieren bzw. zweckmäßige Modifikationen/Ausbaumöglichkeiten vorzuschlagen.

Die KMU FORSCHUNG AUSTIRA hat für die Durchführung der Evaluierung auf einen Mix auf quantitativen und qualitativen Methoden zurückgegriffen, die im folgenden Abschnitt 2 näher beschrieben werden. In Abschnitt 3 erfolgt eine genauere Beschreibung des Evaluierungsgegenstandes, bevor in Abschnitt 4 die Programmabwicklung und in Abschnitt 5 die Mobilisierungswirkungen des ImpulsProgramm creativwirtschaft näher analysiert werden. Abschnitt 6 untersucht daraufhin die Wirkungen des Programms auf der Projektebene und Abschnitt 7 fasst schließlich die wichtigsten Ergebnisse der Evaluierung zusammen und zieht Schlussfolgerungen in Hinblick auf die Weiterführung des Programms. 


\section{Methodische Vorgehensweise}

Für die begleitende Evaluierung des ImpulsProgramm creativwirtschaft hat die KMU FORSCHUNG AUSTRIA auf einen Mix aus quantitativen und qualitativen Methoden zurückgegriffen, die im Folgenden kurz erläutert werden:

\section{Dokumentenanalyse}

Die Dokumentenanalyse erfolgte über die gesamte Laufzeit der Evaluierung parallel zu den weiteren Evaluierungsschritten. Bestandteil der Dokumentenanalyse waren in erster Linie die vom Auftraggeber zur Verfügung gestellten Programmdokumente (Richtlinien, Status- und Tätigkeitsberichte, Jurybesetzungen, Flyer und Folder des Programms sowie der Begleitmaßnahmen, Zeitpläne etc.).

\section{Workshops und Zwischenbesprechungen}

Insgesamt wurden im Rahmen der gegenständlichen Evaluierung neben dem Kick-off Meeting zu Beginn des Projektes, das in erster Linie dazu diente, bestehende Erwartungen und Anforderungen an die Evaluierung zu erfassen und abzustimmen, 2 Workshops bzw. Zwischenbesprechungen durchgeführt. Ein wesentliches Element stellte dabei der Logic Chart Workshop dar, in dem gemeinsam mit der Programmleitung von iP die Funktions- und Wirkungsweise der einzelnen Bestandteile des iP Programms diskutiert und eine ausführliche Zielanalyse des Programms vorgenommen wurde.

\section{Standardisierte Online-Befragung}

Um eine umfassende Darstellung und Bewertung der Aktivitäten von iP aus dem Blickwinkel der einreichenden Unternehmen zu erhalten, wurde eine standardisierte quantitative Erhebung in Form einer Online-Befragung durchgeführt. Befragt wurden alle erfolgreichen Einreicher/innen des ersten (24 Betriebe) und zweiten Calls (16 Betriebe) sowie sämtliche Einreicher/innen des zweiten und dritten Calls (266 Unternehmen). Insgesamt wurden somit 306 Unternehmen kontaktiert, wovon sich 90 an der OnlineBefragung beteiligten. Dies entspricht einer Rücklaufquote von knapp 30\%. Tabelle 1 gibt die Rücklaufquoten in den einzelnen Bereichen wieder.

Tabelle 1 Rücklaufquoten der Online-Befragung der an iP teilnehmenden Unternehmen, nach Call und nach Förderempfänger/Einreicher

\begin{tabular}{l|c|c|c}
\hline & $\begin{array}{c}\text { Ausgesendete } \\
\text { Fragebögen }\end{array}$ & $\begin{array}{c}\text { Ausgefüllte } \\
\text { Fragebögen }\end{array}$ & Rücklaufquote in \% \\
\hline $\begin{array}{l}\text { 1. Call } \\
\text { Förderempfänger }\end{array}$ & 24 & 19 & 79,2 \\
$\begin{array}{l}\text { 2. Call } \\
\text { Förderempfänger }\end{array}$ & 16 & 14 & 87,5 \\
$\begin{array}{l}\text { 2. und 3. Call } \\
\text { Einreicher }\end{array}$ & 266 & 57 & 21,4 \\
\hline \hline & 306 & 90 & 29,4 \\
\hline
\end{tabular}




\section{Interviews mit relevanten Stakeholdern}

Ergänzend zu den quantitativen Evaluierungsergebnissen auf Basis der Unternehmensbefragung wurden insgesamt 16 qualitative Interviews mit Programmverantwortlichen, in iP eingebundenen Personen, Jurymitgliedern, Mitgliedern des iP Beirats, Expert/innen aus der Kreativwirtschaft sowie Netzwerkpartnern durchgeführt Hierfür wurde ein Interviewleitfaden mit offenen halbstandardisierten Fragen verwendet, wobei einzelne Fragestellungen den interviewten Personen und ihrer Rolle im Zusammenhang mit iP angepasst wurden. Die Interviews wurden nach Maßgabe der Erreichbarkeit persönlich oder telefonisch durchgeführt. (Für eine detaillierte Liste der im Rahmen der Evaluierung interviewten Personen, siehe Anhang) 


\section{Beschreibung des Evaluierungsgegenstandes}

\subsection{Eckpunkte des Programms}

Beim ImpulsProgramm creativwirtschaft der aws handelt es sich um ein thematisch orientiertes Förderprogramm, das auf den Bereich der Kreativwirtschaft fokussiert. Das Programm besteht, nach einem entsprechenden Beschluss der Nationalstiftung für Forschung, Technologie und Entwicklung, seit dem Jahr 2004. Das ImpulsProgramm creativwirtschaft war mit departure (Förderstelle der Stadt Wien für Wiener Unternehmen aus der Kreativwirtschaft) eines der ersten Programme, die sich mit der Förderung von Unternehmen aus der Kreativwirtschaft befassen. In den letzten Jahren sind zahlreiche regionale Initiativen zur Förderung der Kreativwirtschaft hinzugekommen (wie z. B. in der Steiermark die neu gegründete Creative Industries Styria $\mathrm{GmbH}$ ). Jedoch stellt das ImpulsProgramm das einzige derartige bundesweite Förderprogramm dar. Weitere Charakteristika des Programms sind das bis dato verwendete Auswahlverfahren über Calls sowie die Einschränkung der Ausschreibung auf die Bereiche Multimedia, Design und Musik.

Das Programm hat keine definierte Laufzeit - die Finanzierung (und somit die Programmfortführung) wird jährlich per Beschlussfassung festgesetzt. Für die Durchführung der ersten beiden Calls (1. Call: 2004/2005, 2. Call: 2005/2006) wurden Stiftungsmittel im Ausmaß von insgesamt $€ 4,8$ Mio ( $€ 2,1$ Mio für den 1. Call, $€ 2,7$ Mio für den 2. Call) bereitgestellt. Für den 3. Call, der 2007 stattgefunden hat, betrugen die Stiftungsmittel $€ 2,5$ Mio.

Von 2004 bis 2006 war das Büro des Programms im Museumsquartier in Wien angesiedelt. Träger der Initiative waren die Wirtschaftskammer Österreich (arge creativwirtschaft austria) sowie die Austria Wirtschaftsservice GesmbH (aws). Im November 2006 wurde das Programm in das Leistungsportfolio der aws eingegliedert; im Zuge dessen erfolgte außerdem auch die Übersiedlung des Programmbüros in die Räumlichkeiten der aws. Die arge creativwirtschaft hat seit dieser Eingliederung in die aws die Rolle eines Kooperationspartners bzw. eines strategischen Partners übernommen und ist somit seitdem nicht mehr Ko-Träger des Programms.

Diese organisatorische Änderung ist insgesamt positiv zu beurteilen. Die vollständige Eingliederung in die aws ermöglicht die Nutzung von Synergiepotenzialen zu anderen Förderprogrammen und trägt dazu bei, die Förderabwicklung effektiver und effizienter zu gestalten. Die Effizienzsteigerung ergibt sich dabei - so die einstimmige Meinung der involvierten Akteure - vor allem aus den kürzeren Informationswegen zwischen dem Programmmanagement (das mit den Besonderheiten der Kreativwirtschaft vertraut ist und diese nun schneller/direkter vermitteln kann) und den handelnden Akteuren in der Förderabwicklung im „Back Office“ Bereich. Eine gewisse Herausforderung besteht seit der Eingliederung jedoch darin, das eigenständige Auftreten des ImpulsProgramm creativwirtschaft aufrecht zu erhalten, was insbesondere in Hinblick auf die Zielgruppenansprache - vor dem Hintergrund, dass sich viele in den Cl tätige Personen nicht als Wirtschaftstreibende sehen und daher oftmals eine gewisse Distanz zu wirtschaftsorientierten Fördereinrichtungen wie der aws haben - von den interviewten Expert/innen als essentiell angesehen wird (siehe auch Kapitel 5.3). 
Das iP Team, das aus drei Personen besteht, steht seit dem Beginn des Programms unter der Leitung von Frau Mag. Pümpel. Drei Expert/innen aus den jeweiligen Bereichen Musik, Multimedia und Design wurden ernannt, um das Projektteam auf inhaltlicher Ebene beratend zur Seite zu stehen. Weiters wurde 2007 ein Kreativwirtschaftsbeirat einberufen, der sich u.a. aus Vertreter/innen der WKO, der aws, des BMWA, des Rats für Forschung und Technologieentwicklung sowie Kreativwirtschaftsexpert/innen zusammensetzt. Der Beirat berät das ImpulsProgramm betreffend Maßnahmen und Förderungsmöglichkeiten im Kreativwirtschaftsbereich. Er soll außerdem als Plattform für aktiven Wissensaustausch zwischen den teilnehmenden Organisationen zum Thema Kreativwirtschaft dienen.

Auf regionaler Ebene kooperiert das ImpulsProgramm mit zahlreichen Netzwerkpartnern aus den Bereichen Kreativwirtschaft und Technologieförderung. Die Kooperation zielt auf die Bewerbung des Programms in den Bundesländern durch die Netzwerkpartner ab. Stärker involvierte bzw. motivierte Netzwerkpartner können eine aktivere Rolle einnehmen, z. B. als Informations- und Beratungsstelle, wie dies z.B. bei einem Netzwerkpartner in Tirol für 2008 geplant ist. Das CAST Tyrol soll in Zukunft die Einreichberatungen für iP für potenzielle Einreicher aus den westlichen Bundesländern übernehmen.

\subsection{Ziele und Inhalte des Programms}

Mit Hilfe der Logic Chart Methode, die im Rahmen von moderierten Workshops zum Einsatz kommt, wird versucht die Funktionsweise eines Förderprogramms, die Programmlogik, ausgehend von den Zielen, über die eingesetzten Ressourcen, die Implementierung bis hin zu den (tatsächlichen wie gewünschten) Ergebnissen möglichst übersichtlich in einem Flussdiagramm darzustellen. Dadurch ergibt sich der Vorteil, dass die wesentlichen Elemente des Programms und dessen Wirkungen auf einer Seite dargestellt werden können. Für die vorliegende Evaluierung wurde auf Basis der entsprechenden Programmdokumente gemeinsam mit dem Programmmanagement untenstehender Logic Chart entwickelt (siehe Grafik 1).

Die Programmlogik findet zunächst ihren Anfangspunkt in der Mission des Programms, die als Globalziel der Initiative angesehen werden kann. Diese „Mission“ liegt im Kontext des ImpulsProgramm creativwirtschaft in erster Linie in der "Steigerung des Innovationspotenzials und der Wettbewerbsfähigkeit von KMU in und mit Hilfe der Bereiche Musik, Multimedia und Design" sowie in der "Stärkung des Kreativstandortes Österreich und dessen internationale Positionierung". Zur Erreichung dieses Globalziels wurden in einer ersten operativen Ausdifferenzierung zwei Subziele definiert: So sollen einerseits mehr Innovationen durch mehr kreative Leistungen geschaffen werden, zum Anderen soll die wirtschaftliche Kompetenz der Unternehmen in den kreativen Branchen gesteigert werden. 


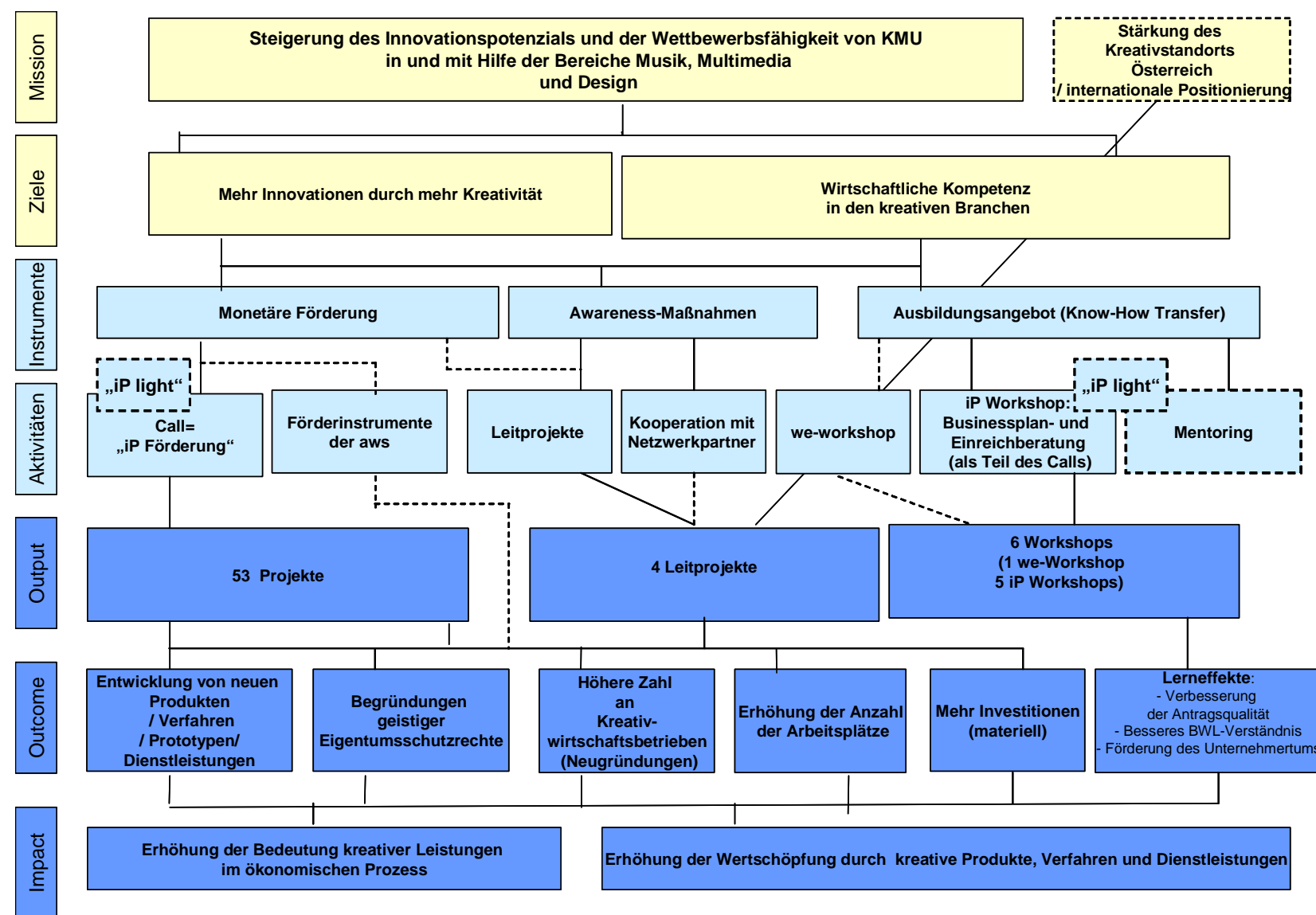

iP Light: Mögliche zukünftige Positionierung von einer „iP Light“ Programmschiene (siehe hierzu auch Abschnitt 5.1) Quelle: KMU FORSCHUNG AUSTRIA 
Zur Erreichung der Ziele des ImpulsProgramm creativwirtschaft wird auf insgesamt drei Förderinstrumente zurückgegriffen:

- Eine monetäre Förderung (die sogenannte „iP Förderung“) für kreative, innovative und marktorientierte Projekte mit Kreativwirtschaftsbezug;

- Bewusstseinsbildende Maßnahmen, die dazu beitragen sollen, einerseits das wirtschaftliche Potenzial bei kreativ tätigen Personen in Österreich zu mobilisieren, andererseits aber auch die Bekanntheit von iP bei der Zielgruppe und in der Öffentlichkeit zu erhöhen;

- Ein Ausbildungsangebot, das im Wesentlichen aus einer Reihe von auf die $\mathrm{Cl}$ zugeschnittenen Workshops besteht.

Im Folgenden werden die einzelnen Instrumente näher beschrieben.

\section{Die iP Förderung}

Bei der monetären Förderung handelt es sich um die „iP Förderung“ im engeren Sinn, d. h. um einen monetären Zuschuss (bis zu $70 \%$ der förderbaren Projektkosten) für kreative, innovative und marktorientierte Projekte (Produkte, Verfahren, Dienstleistungen) aus den Kreativbereichen Musik, Multimedia und Design. Die Auswahl der förderbaren Projekte erfolgt über die Durchführung von Calls. Gefördert werden Projekte, die nicht nur eine hohe Relevanz für den jeweiligen Kreativbereich haben sondern auch eine erfolgreiche wirtschaftliche Umsetzung erwarten lassen bzw. einen Wertschöpfungsprozess generieren.

Die Calls werden online durchgeführt. Das bedeutet, dass die Einreicher über ein Login verfügen, das innen den Zugang zu ihren Antragsunterlagen ermöglicht. Für die Projektauswahl wird dabei auf ein zweistufiges Verfahren zurückgegriffen. In der ersten Phase müssen die Einreicher einen Antrag stellen, in dem die wichtigsten Kenndaten des Unternehmens und des Projekts erläutert werden. Diese Anträge werden zuerst wirtschaftlich von der aws-Jury und dann inhaltlich von einer Fachjury zunächst online und anschließend im Rahmen einer Jurysitzung (des jeweiligen Themenbereichs) bewertet. Jene Antragsteller, die zur Phase 2 zugelassen werden, sind eingeladen, ihre Projektideen detaillierter auszuarbeiten und einen Businessplan zu erstellen. Danach erfolgt wiederum eine wirtschaftliche Bewertung durch die aws und eine inhaltliche online-Bewertung durch die Fachjury. Die Förderentscheidung wird letztlich im Rahmen der Jurysitzung getroffen, an der Vertreter/innen der Fachjury, der aws-Jury und Expert/innen aus dem Beirat teilnehmen.

Es ist von der Programmleitung geplant, das Antragsystem in Zukunft zu verändern. Anstelle des zweiphasigen Antragsystems soll ab dem 4. Call, d.h. ab 2008, nur mehr eine einzige Antragstellung notwendig sein. Die Antragsteller werden einen Antrag ausfüllen müssen, der Elemente aus den bestehenden beiden Antragstellungsphasen beinhaltet. Nach der Antragstellung sollen die ausgewählten Projekte nach 4 Wochen zu einem Hearing vor die Fachjury eingeladen werden. Der Grund für die geplante Umstellung liegt in der langen Dauer des bestehenden Callverfahrens. Anstelle einer Einreichung pro Jahr sollen dadurch in Zukunft zwei Termine ermöglicht werden. Das ist gemäß Expert/innen u. a. angesichts der kurzen „time-to-market“ Zeit von Innovationsprojekten insbesondere in der Kreativwirtschaft als durchaus positiv zu werten. Dem ist allerdings auch in Rechnung zu stellen, dass es gemäß Meinungen der befragten Expert/innen insbesondere dem aufwendigen Auswahlverfahren (unter Ein- 
beziehung einer adäquaten Anzahl internationaler Juror/innen) zu verdanken ist, dass sich das Förderprogramm in der Zielgruppe eine hohe Reputation aufbauen konnte. Wie später gezeigt wird, ist in der Reputation des Programms ein wesentlicher Erfolgsfaktor zu sehen (siehe hierzu Kapitel 5) Es sollte daher angestrebt werden, die Größe sowie v. a. die internationale Zusammenstellung der Jury, die die Projekte bewertet, nicht zu verändern, um Qualitätsverluste im Auswahlverfahren zu vermeiden.

\section{Das Ausbildungsangebot}

Das Ausbildungsangebot von iP beinhaltet einerseits eine wirtschaftliche Weiterbildung (darunter fallen die we-workshops und der Businessplan-Workshop) und andererseits die Unterstützung bei der Projektdarstellung (von iP angebotene Einreichberatung). Die Workshops „iP Businessplan“ sowie „iP Einreichberatung“ werden im Rahmen des 2. und 3. Calls angeboten. Einreicher, die im zweistufigen Auswahlverfahren in die Phase 2 gelangten, konnten an diesen Workshops teilnehmen. Der we-Workshop wird in Kooperation mit departure, der Förderstelle für Kreativwirtschaft der Stadt Wien, angeboten und ist eine von der monetären Förderung unabhängige Workshopschiene. Es werden darin Basis- und Praxiswissen von Branchenkennern für die erfolgreiche Gründung eines Unternehmens in der Kreativwirtschaft vermittelt.

\section{Bewusstseinsbildende Maßnahmen}

Mit den Awareness-Maßnahmen sollen der Wert und das Potenzial kreativer Leistungen in der Öffentlichkeit erhöht und wirtschaftliches Potenzial im Sinne des Fördertatbestandes von iP mobilisiert werden. Netzwerkpartner aus allen österreichischen Bundesländern dienen dabei unter anderem als Multiplikatoren. Bei den regionalen Partnern handelt es sich einerseits um Institutionen aus dem Kreativwirtschaftsbereich (z. B: die Ars Electronica, das Netzwerk für Design und Medien) und andererseits um Fördereinrichtungen für Unternehmen aus der Kreativwirtschaft (z. B: SFG Kreativwirtschaft, Musikfonds, departure). Zu den Awareness-Maßnahmen im Kontext von iP können außerdem die Leitprojekte gezählt werden. Diese branchenspezifischen Leitprojekte erhalten einen finanziellen Zuschuss durch das ImpulsProgramm und können selbst als regional- und/oder Cl-themenspezifische Förderprogramme angesehen werden. Bislang wurden vier derartige Leitprojekte initiiert: AMAN - Austrian Music Ambassador Network, das Designforum in Wien, die Initiativen „Kreatives Handwerk Salzburg“ und das „Kreative Handwerk Tirol“. Für 2008 sind weitere Leitprojekte geplant.

Insgesamt ergibt sich das Bild, dass sich das Programm seit dem 1. Call im Jahr 2004/2005 in weiten Teilen nicht nur organisatorisch sondern auch inhaltlich weiterentwickelt hat, speziell mit Blickpunkt auf das Begleitprogramm. Zum Einen wurden die „we-Workshops" gemeinsam mit der Wiener Förderstelle departure konzipiert.' Zum Anderen wurden auch das Angebot an Einreichberatungs- und Businessplanworkshops systematisch für alle Unternehmen, die für die 2. Phase des Antragverfahrens zugelassen wurden, erweitert. Als weitere Veränderung bzw. Schwerpunktsetzung erfolgte die Intensivierung der Zusammenarbeit mit regionalen Förderstellen und Netzwerkpartnern (It. Programmdokument und Aktivitätenplan 2007) sowie die Schaffung des Expert/innenbeirats (siehe Kapitel 3.1) und die Adaption der Richtlinien und Flyer-

\footnotetext{
Die Interviewresultate deuten darauf hin, dass die Zusammenarbeit zwischen departure und iP in den Überlappungsbereichen der beiden Programme bislang durch realisierte Synergiepotenziale geprägt ist - weitere Belege hierfür sind neben den gemeinsam organisierten we workshops auch häufiger stattfindende gegenseitige Konsultationen (z.B. über die wechselseitige Mitwirkung in Programmberatungs-/aufsichtsgremien).
} 
bzw. Homepagetexte, die mittels der gewonnenen Erfahrungen und Ergebnisse der ersten Zwischenevaluierung angepasst wurden.

In Summe führten die durchgeführten Adaptionen im Programmdesign u. a. zu einer deutlich verbesserten Handhabung des Spannungsfeldes zwischen „technischer Innovationsförderung“ und „Förderung kreativ-künstlerischer Leistungen“, einer in der ersten Zwischenevaluierung deutlich angesprochenen Schwachstelle des Förderprogramms. Diese bessere Handhabe spiegelt sich laut Juror/innen auch in den Projektanträgen wider:

„Man sieht bei den Einreichungen, dass zwar innovative Technologien im Einsatz sind, aber nicht nur Technologieentwicklung passiert, sondern wirklich der Business Case, die Idee wichtiger wird. Das war früher nicht so." (Juror)

Eine Entwicklung, die in Hinblick auf die Zielsetzung des ImpulsProgramm creativwirtschaft auch aus Sicht der Evaluator/innen durchaus begrüßenswert ist. 


\section{$4 \quad$ Analyse der Programmabwicklung}

Im Folgenden steht zunächst die Abwicklung des Förderprogramms im Vordergrund der Betrachtungen. Insbesondere werden die Zufriedenheit der Förderwerber mit verschiedenen Aspekten der Abwicklung und daraus resultierende Implikationen für das Programmmanagement erörtert. Zu diesem Zweck erfolgt eine Unterteilung in die Bereiche „generelle Programmabwicklung" und „Callabwicklung", um zwischen allgemeinen Programmmanagementtätigkeiten und Call-spezifischen Aktivitäten differenzieren zu können, sowie eine weitere Abgrenzung zu den Begleitmaßnahmen.

\subsection{Generelle Programmabwicklung}

Die befragten Unternehmen sind mit der generellen Programmabwicklung sehr zufrieden (siehe Grafik 2). Die Organisation und Gestaltung von Informationsveranstaltungen, der Umfang und die Klarheit der Informationsunterlagen, die Übersichtlichkeit und der Informationsgehalt der Homepage sowie die Beratung und Betreuung der iP-Mitarbeiter/innen und deren Erreichbarkeit wurden mit Durchschnittswerten von 2,0 (auf einer Skala von 1 = sehr zufrieden bis 4 = nicht zufrieden) oder besser durchwegs hervorragend bewertet. Die im Vergleich schlechteste - aber immer noch gute - Bewertung von durchschnittlich 2,0 erhielt die Homepage des Programms, was evtl. damit zusammenhängen könnte, dass die Homepage nun Teil der aws Website ist. Dem ist allerdings auch in Rechnung zu stellen, dass die Domain der Homepage (http://www.impulsprogramm.at) ebenso erhalten geblieben ist wie das iP Logo. Von den interviewten Expert/innen wurde mehrfach betont, dass eine Beibehaltung der Marke iP für die Zielgruppenakzeptanz von hoher Signfikanz ist (siehe auch Kapitel 5.3).

Grafik 2 Zufriedenheit mit Aspekten der generellen Programmabwicklung, auf einer Skala von 1= sehr zufrieden bis 4= gar nicht zufrieden, arithmetische Mittelwerte der Antworten der einreichenden Betriebe aus dem 2. und 3. Call

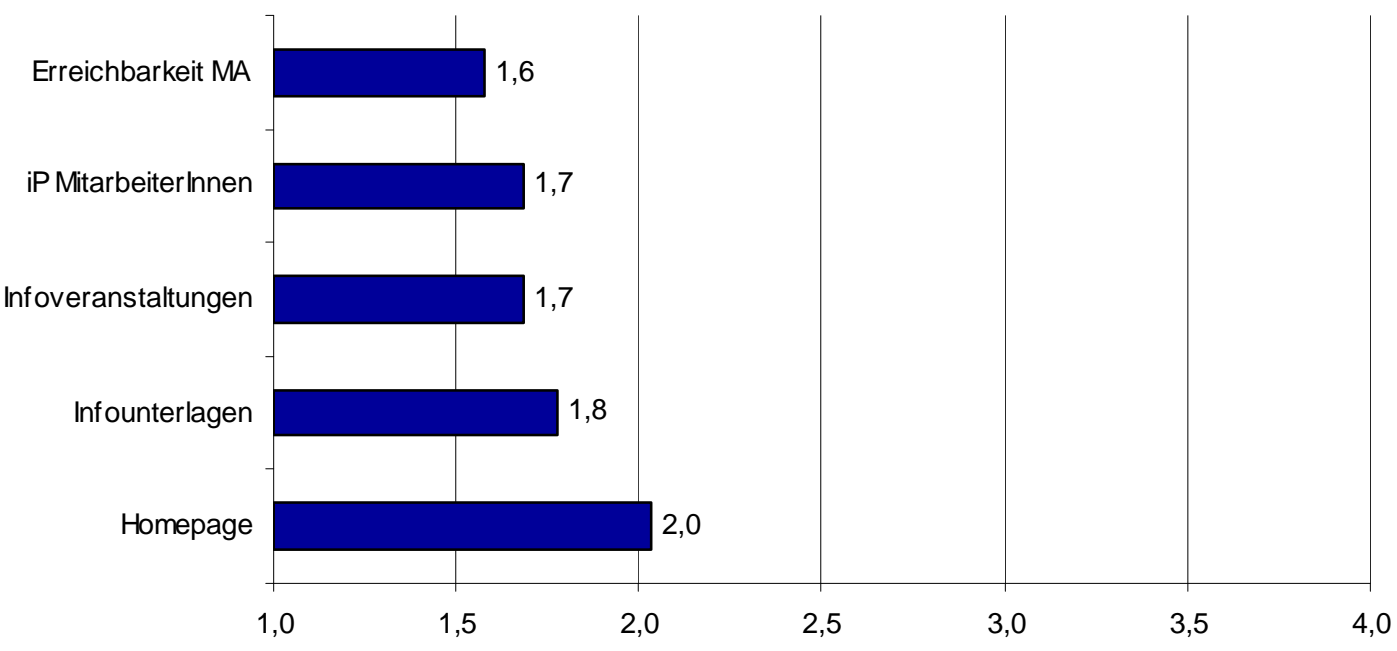

Quelle: KMU FORSCHUNG AUSTRIA, n=62 
Das Engagement der iP Mitarbeiter/innen wird, außer von den befragten Förderwerbern, auch von den interviewten Juroren/innen und Expert/innen deutlich hervorgehoben, insbesondere was die gemeinsam mit den involvierten Expert/innen aus der Kreativwirtschaft erfolgte Erarbeitung von Konzepten für die operative und strategische Weiterentwicklung des Förderprogramms betrifft. Die pro-aktive Herangehensweise des iP Teams und die intensive Kommunikation zwischen Beirat, iP Team und involvierten Expert/innen dürfte maßgeblich mitverantwortlich dafür sein, dass das Programm sich in der beobachteten Geschwindigkeit weiterentwickelt hat und auch weiterhin Verbesserungen und Adaptierungen zeitnah in das ImpulsProgramm einflieBen.

\subsection{Callabwicklung}

Die in Abschnitt 4.1 dargestellten insgesamt begrüßenswerten intensiven Austauschund Kommunikationsflüsse bergen, wie im Folgenden gezeigt wird, in einer speziellen Konstellation, nämlich im Zusammenspiel mit der Handhabung von Förderabsagen, auch die Gefahr einer potenziell schädlichen Lock-in Situation. ${ }^{2}$ Grafik 3 zeigt hierfür zunächst die Zufriedenheit der Förderwerber mit verschiedenen Aspekten der Antragsstellungsphase im Zuge der Callabwicklung.

Zunächst wird deutlich, dass die Beurteilung der Antragsstellungsphase in der Callabwicklung deutlich differenzierter ausfällt als jene der generellen Programmabwicklung. Was die Unterstützungsleistung bei der Antragstellung in Phase 2, die Zeit, die zur Erstellung eines Antrags zur Verfügung steht, die Verständlichkeit der Ausschreibungsunterlagen und die Klarheit der Antragsformulare betrifft, erfolgte eine sehr gute bis gute Bewertung. Die Beurteilung des administrativen Aufwands bei der Antragstellung in Phase 1 und 2 sowie der Bearbeitungsdauer des Projektsantrags fiel mit einer „Durchschnittsnote“ von 2,3 bzw. 2,4 etwas schlechter aus - hier ist aber auch in Rechnung zu stellen, dass ein gewisser administrativer Aufwand bei Förderprogrammen unumgänglich ist.

Unzufriedenheit herrscht bei den einreichenden Unternehmen des 2. und 3. Calls bezüglich der Transparenz der Beurteilungskriterien und dem Feedback zu den Ablehnungsgründen nach Phase 1. Die negative Bewertung dieser beiden Aspekte ist bei der Zwischenevaluierung des 1. Calls schon aufgefallen (vgl. Grafik 3).

\footnotetext{
Unter einer Lock-in Situation soll im vorliegenden Kontext der Sachverhalt verstanden werden, wonach nach außen hin die Wahrnehmung einer in sich weitgehend geschlossenen Gruppe von Mitarbeiter/innen des iP Teams und einer Zahl von iP Expert/innen erzeugt wird, wo zwar innerhalb der Gruppe intensiv kommuniziert wird bzw. Konzepte erarbeitet werden (und auch die Überzeugung über die gute Arbeit innerhalb der Gruppe vorherrscht), inhaltlich gute Ideen und Vorstellungen über förderbare Projekte, die außerhalb dieses Kreises generiert werden, aber (scheinbar oder nicht) nur schwer Eingang in das Förderprogramm finden.
} 
Grafik 3 Zufriedenheit mit Aspekten der Antragstellungsphase, auf einer Skala von $1=$ sehr zufrieden bis $4=$ gar nicht zufrieden, arithmetische Mittelwerte der Antworten der einreichenden Betriebe aus dem 2. und 3. Call

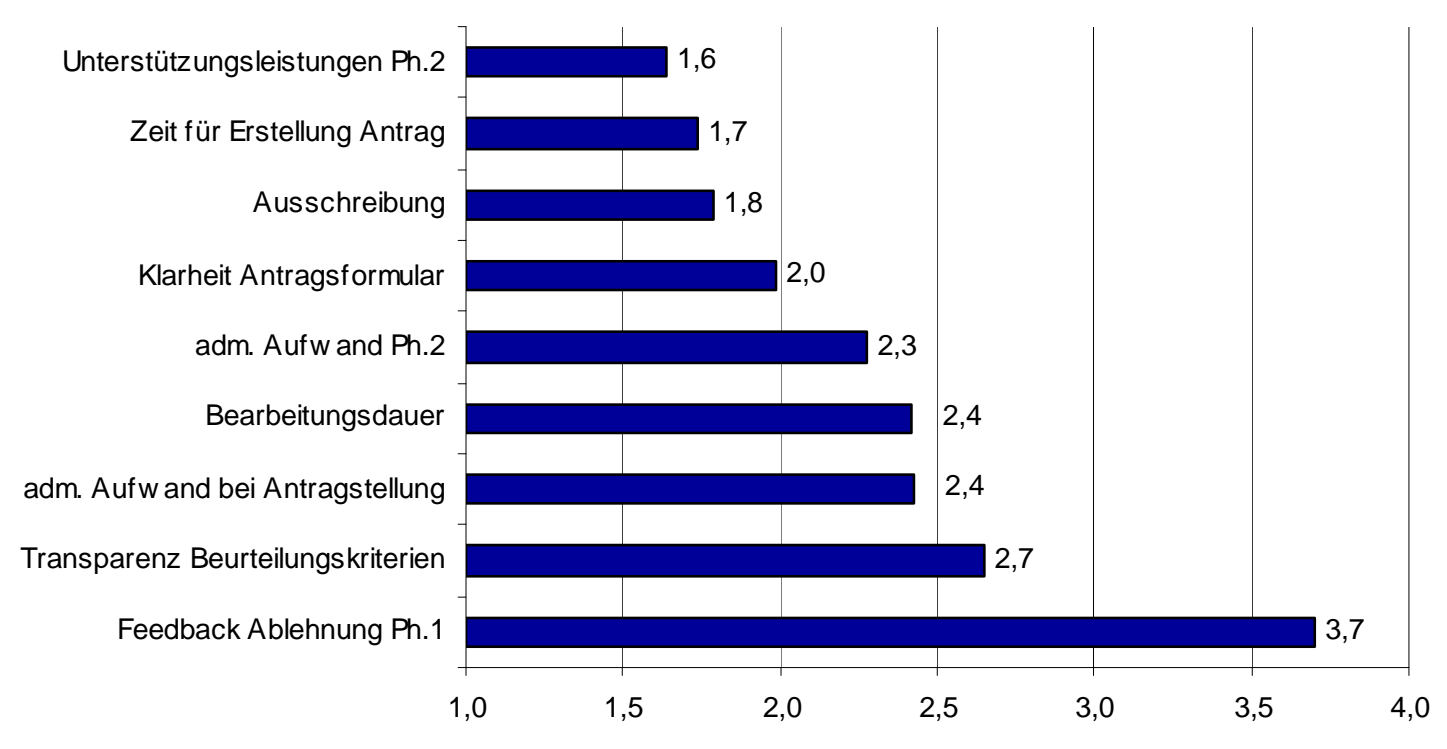

Quelle: KMU FORSCHUNG AUSTRIA, $n=64$

Zweifellos ergibt sich bei Aspekten wie Feedback zu Ablehnungsgründen immer eine Art Sockelunzufriedenheit auf Grund der Enttäuschung über den nicht erfolgreichen Antrag; zudem ist hinsichtlich der Transparenz der Beurteilungskriterien besonders bei der Beurteilung von kreativen Aspekten immer ein subjektiver Anteil nicht zu vermeiden. Aus den Interviewergebnissen lassen sich aber auch Faktoren im ImpulsProgramm creativwirtschaft identifizieren, die das Potenzial haben, eine derartige Unzufriedenheit zu verstärken.

Die genannten intensiven Kommunikationsbeziehungen zwischen den Mitgliedern des iP Teams und engagierten $\mathrm{Cl}$ Expert/innen sind ein erster derartiger Faktor: Sie könnten in der Außenwahrnehmung den Eindruck von Abschottungstendenzen erkennen lassen, wie eine Juror/in festgestellt hat:

\section{„Von außen wirkt das Programm wie ein Insider-Klub.“(Juror/in)}

Ein zweiter Faktor ergibt sich aus der hohen Selektivität der Förderung, die mitunter bewirken kann, dass auch gute Projekte keine Förderzusage erhalten (weil es noch bessere Anträge gibt). Geht der Antrag in der zweiten Runde leer aus - zu einem Zeitpunkt, zu dem bereits viel Arbeit in die Antragserstellung geflossen ist, evtl. inklusive einem Besuch von Begleitworkshops -, ist die Enttäuschung wahrscheinlich noch viel größer, insbesondere wenn das Projekt gut genug ist, an anderer Stelle doch mit einem Preis oder einer Förderung bedacht zu werden. In diesem Fall ist der Wunsch nach einer Begründung für die Ablehnung besonders ausgeprägt. So meinte etwa ein Einreicher in dieser Situation: 
„ICh wünsche mir eine "sinnvolle" Begründung der Ablehnung - "kein Innovationsgrad" als Ablehnungsgrund stellt keine Aussage dar, die dem Einreicher weiterhilft. Wenn mein Projekt nicht innovativ wäre, wäre ich nicht zweimal zur [bedeutende Veranstaltung in der Branche, in der der Einreicher tätig ist] eingeladen worden. Das Urteil der Jury kann dem Einreicher wertvolle Unterstützung sein." (Einreicher)

Ein anderer Einreicher äußerte sich in ähnlicher Weise mit den Worten

„...ich fühle mich wirklich gelinkt"

sichtlich verärgert über die (fehlende bzw. als unzureichend angesehene) Begründung der Ablehnung seines Projektantrages. Es ist allerdings anzumerken, dass die Kombination der genannten Faktoren (durch die Antragsteller wahrgenommene Abschottungstendenzen von iP nach außen hin; als unzureichend empfundene Begründung der Ablehnung des Projektantrags; möglicherweise Evidenzen, dass das Projekt dennoch von durchaus hoher Qualität war/ist) im Pool der abgelehnten Anträge nur selten zu beobachten war. Der Fördergeber sollte sich nichtsdestotrotz der latenten (vor allem wegen der Subjektivitätsproblematik bei der Beurteilung von Kreativleistungen) Problematik eines möglicherweise in den Augen von potenziellen guten Förderwerbern operierenden „Insider“-ImpulsProgramm bewusst sein insbesondere bei der Kommunikation von Ablehnungsgründen sollte nicht auf Grund des administrativen Aufwandes gespart werden. Die bereits eingeführte Praxis, telefonisch auf Anfrage detailliert Auskunft zu geben, ist in dieser Hinsicht zu begrüßen; insbesondere gilt dies für das Vorhaben, dass Einreicher ihre Projekte persönlich vor der Jury verteidigen können, was auch von Förderwerbern explizit gefordert wird:

„...besser wäre [eine] Vorstellung der Projekte in Phase2 persönlich! Die für den Antragsteller anonyme Jury sieht sehr "willkürlich" aus!" (Einreicher)

„...Als Designer habe ich keine Schwierigkeiten kreative Produkte zu entwickeln! Ich habe Schwierigkeiten die Umsetzung zu finanzieren bzw. Firmen zu überzeugen sich an den Kosten der Entwicklung zu beteiligen. Ich verstehe dann z.B. nicht wieso bei einem Förderprogramm die Kreativität des Produktes beurteilt wird und das auch von Personen die weniger Fachwissen aufbringen als ich. Eine solche Beurteilung sollte auf jeden Fall in einem persönlichen Gespräch stattfinden, um Missverständnisse zu klären und nicht bei Kaffee und Kuchen juryintern, da es sich bei den meisten Antragstellern um die Zukunft des Unternehmens handelt...." (Einreicher)

Weitere Ansatzpunkte könnten Internetforen und Blogs sein, die zudem ein kostenmäßig geringeres Risiko aufweisen, oder auch eine Intensivierung der Juror/innenrotation. Sog. „learning-loops“ auf Basis eines Monitorings der geförderten Projekte im Zeitverlauf könnten in diesem Zusammenhang beispielsweise dazu beitragen, Lerneffekte auf Ebene der Juroren/innen zu generieren („haben wir das Projekt im Rahmen des Calls gut ausgesucht? Ist das Unternehmen mit dem Projekt erfolgreich? Haben 
wir die richtigen Auswahlkriterien angewendet?") und somit die Qualität und Transparenz des Auswahlverfahrens laufend zu erhöhen. ${ }^{3}$

Die Abwicklung der Förderung nach erfolgter Förderzusage durch die aws - d.h. die Auszahlungsmodalitäten und die Betreuung der geförderten Projekte, wird, wie die allgemeine Programmabwicklung, von den Förderempfängern des 1. und 2. Calls weitgehend positiv bewertet. Auffallend ist hier jedoch, dass der administrative Aufwand in der Förderabwicklungsphase sowie die sonstige Abwicklung durch die aws nach der Förderzusage - wenngleich beide als zufriedenstellend beurteilt - in ihrer Bewertung doch hinter die anderen Aspekte, wie etwa die Zufriedenheit mit der Qualität der Beratung und Betreuung durch die aws-Mitarbeiter/innen oder die Erreichbarkeit der aws-Mitarbeiter/innen zurückfallen (vgl. Grafik 4). In Kommentaren wurde vereinzelt ein gewisser Unmut darüber geäußert, dass die Auszahlungsmodalitäten eine Vorfinanzierung erfordern, der „unnötigen Aufwand (Zinsen)" (Zitat Einreicher) für etwaig in Anspruch genommene Kreditfinanzierungen nach sich ziehen soll.

Grafik 4 Zufriedenheit mit Aspekten der Förderabwicklung, auf einer Skala von 1= sehr zufrieden bis 4= gar nicht zufrieden, arithmetische Mittelwerte der Antworten der geförderten Betriebe aus dem 1. und 2. Call

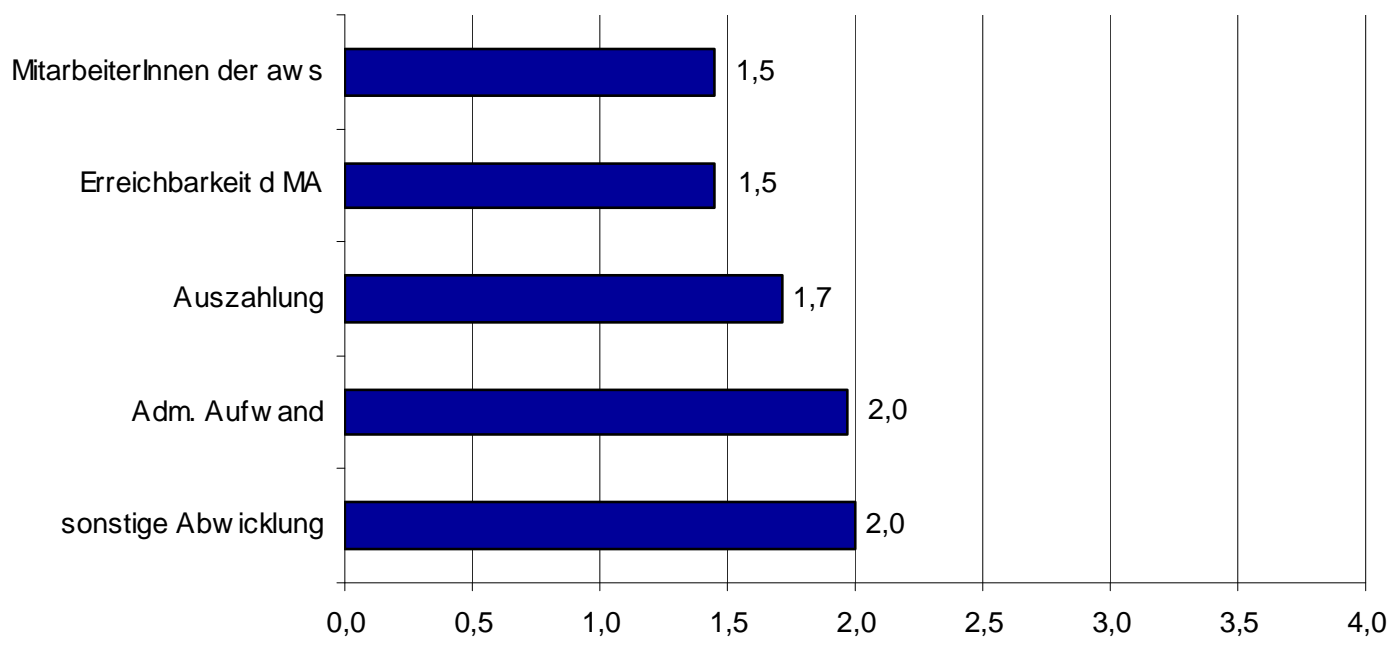

Quelle: KMU FORSCHUNG AUSTRIA, $n=31$

Bei allen angezeigten Zufriedenheitswerten waren signifikante Unterschiede in den Zufriedenheitsniveaus nach Themenbereichen (Musik, Multimedia, Design), nach betrieblichen Tätigkeitsbereichen ( $\mathrm{zu}$ den betrieblichen Tätigkeitsbereichen siehe Grafik 14) oder Betriebsgröße nicht zu beobachten (die Bewertung fiel bei Förderwerbern, deren Anträge abgelehnt wurden, naturgemäß etwas schlechter aus als bei den Förderempfängern).

3 Ein positives Beispiel stellt in diesem Zusammenhang etwa das Monitoringsystem der FFG im Bereich Basisprogramme dar, in dem eine Verknüpfung der Bewertungsindikatoren für die Projektauswahl mit der ex post Evaluierung geförderter Projekte erfolgt. Dabei hat sich beispielsweise gezeigt, dass jene Projekte, die sich im Nachhinein als erfolgreich herausstellen von der FFG auf Basis der wirtschaftlichen Beurteilungskriterien bereits zu Beginn besser bewertet werden, als die nicht erfolgreichen Projekte (bei den technischen Bewertungskriterien ist ein derart eindeutiger Zusammenhang nicht zu erkennen). 


\section{Zusammenfassende Betrachtung}

Insgesamt ist zu unterstreichen, dass der Programmabwicklung von den an iP teilnehmenden Unternehmen ein in weiten Teilen positives Zeugnis ausgestellt wird. Eine Ausnahme stellen diesbezüglich lediglich die Beurteilung der Transparenz der Beurteilungskriterien und das Feedback zu den Ablehnungsgründen des Antragsverfahrens dar. Da bei der Beurteilung von Kreativitätsleistungen immer ein gewisser subjektiver Anteil mitentscheidend ist, werden diese beiden Themenkomplexe wohl zum Teil auch weiterhin eine Herausforderung für Förderprogramme im Kreativwirtschaftsbereich bleiben. Umso wichtiger scheint es jedoch, insbesondere in Hinblick auf die Beurteilungskriterien, eine möglichst transparente Bewertungsbasis zu schaffen und diese auch einer kontinuierlichen Reflexion zu unterziehen (z.B. im Rahmen der dargestellten "learning loops"). In Hinblick auf eine Verbesserung des Feedbacks bei abgelehnten Projektanträgen soll auf den erwähnten Vorschlag von Expert/innen, auf kostengünstige Medien wie etwa Internetforen oder Blogs zurückzugreifen, um z.B. Unterschiede zwischen erfolgreichen und nicht erfolgreichen Anträgen zu kommunizieren bzw. zu diskutieren, an dieser Stelle noch einmal hingewiesen werden. Der dargestellten möglichen "Lock-in"-Situation sollte seitens des Fördergebers hinreichend Aufmerksamkeit geschenkt werden. 


\subsection{Begleitmaßnahmen}

Das ImpulsProgramm creativwirtschaft hat sich seit dem 1. Call im Jahr 2004/2005 deutlich weiterentwickelt. Insbesondere im Jahr 2007 lag der Fokus dabei auf dem Ausbau des Begleitprogramms (siehe Kapitel 3.2). Während es demzufolge im 1. Call nur Workshops zum Thema Businessplanerstellung gab (die zudem weniger stark auf die Besonderheiten und Bedürfnisse der $\mathrm{Cl}$ zugeschnitten waren), wurden in den folgenden beiden Calls jeweils zwei weitere iP Workshops angeboten: der „iP Businessplan“ und die „iP Einreichberatung“. Diese konnten von den einreichenden Unternehmen, die für die 2. Antragsphase zugelassen wurden, kostenlos besucht werden. Seit Beginn des Programms wurden im Rahmen des Ausbildungsangebots insgesamt 6 Workshops durchgeführt.

Die Ergebnisse der Unternehmensbefragung zeigen, dass der Businessplan-Workshop von mehr als der Hälfte der zugelassenen Unternehmen in Anspruch genommen wurde. Die Einreichberatung wurde noch besser besucht: Fast drei Viertel der befragten Unternehmen haben an der Einreichberatung teilgenommen. Die Zufriedenheit mit den Workshops war dabei durchwegs sehr hoch. Rd. $90 \%$ der Teilnehmer/innen beider Workshops waren mit den Businessplan Workshops und den Einreichberatungen sehr oder zumindest eher zufrieden. Mit einem Anteil von mehr als zwei Drittel sehr zufriedener Teilnehmer/innen wurde die Einreichberatung dabei noch besser bewertet als der Businesslan-Workshop mit 44\% sehr zufriedenen Kunden/innen (siehe Grafik 5).

\section{Grafik 5 Inanspruchnahme und Zufriedenheit mit den iP Workshops, Betriebe in \%}

\section{Businessplan-Workshop}

Inanspruchnahme

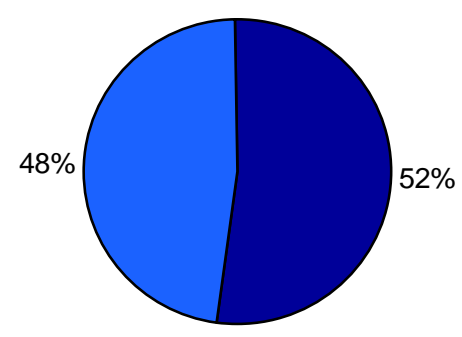

in Anspruch genommen

$\square$ nicht in Anspruch genommen
Zufriedenheit

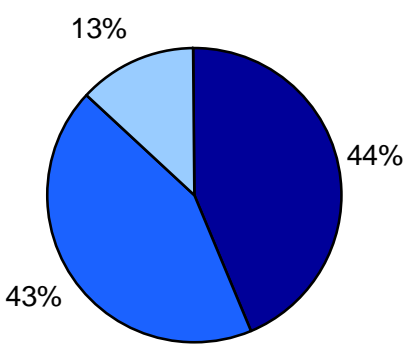

sehr zufrieden $\square$ eher zufrieden $\square$ eher unzufrieden 


\section{Einreichberatung}

Inanspruchnahme

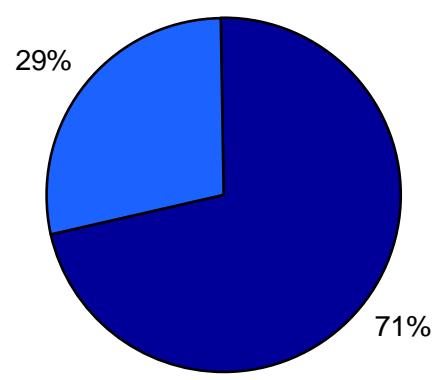

in Anspruch genommen

$\square$ nicht in Anspruch genommen $\mathrm{n}=45$

Quelle: KMU FORSCHUNG AUSTRIA

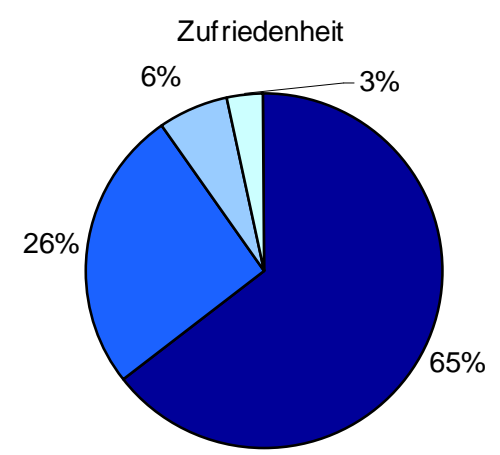

घ sehr zufrieden $\square$ eher zufrieden

$\square$ eher unzufrieden

Die Gründe, warum einige Unternehmen nicht an den angebotenen Workshops teilgenommen haben, sind durchaus vielfältig (siehe Grafik 6). Eindeutig dominierend ist dabei jedoch insbesondere in Hinblick auf die Businessplan-Workshops die Einschätzung der Unternehmen, bereits über die zu vermittelnden Kenntnisse zu verfügen dieser Selbsteinschätzung ist gem. der interviewten Expert/innen aber mit einer gewissen Skepsis zu begegnen. Als weitere wesentliche Gründe für die Nicht-Teilnahme an den angebotenen Workshops wurden der spezifische (zu den angebotenen Terminen) bzw. allgemeine Zeitmangel genannt. Insbesondere mit Blick auf die Einreichberatung dürfte wohl auch noch ein entsprechendes Informationsdefizit bestehen. Einige Unternehmen gaben an, nicht ausreichend über den Workshop informiert gewesen zu sein. In diesem Zusammenhang muss jedoch auch auf die vergleichsweise geringe Fallzahl bei dieser Auswertung hingewiesen werden. 


\section{Grafik 6 Gründe der Nichtteilnahme an den Begleitmaßnahmen von iP,} Betriebe in \%, Mehrfachnennungen möglich

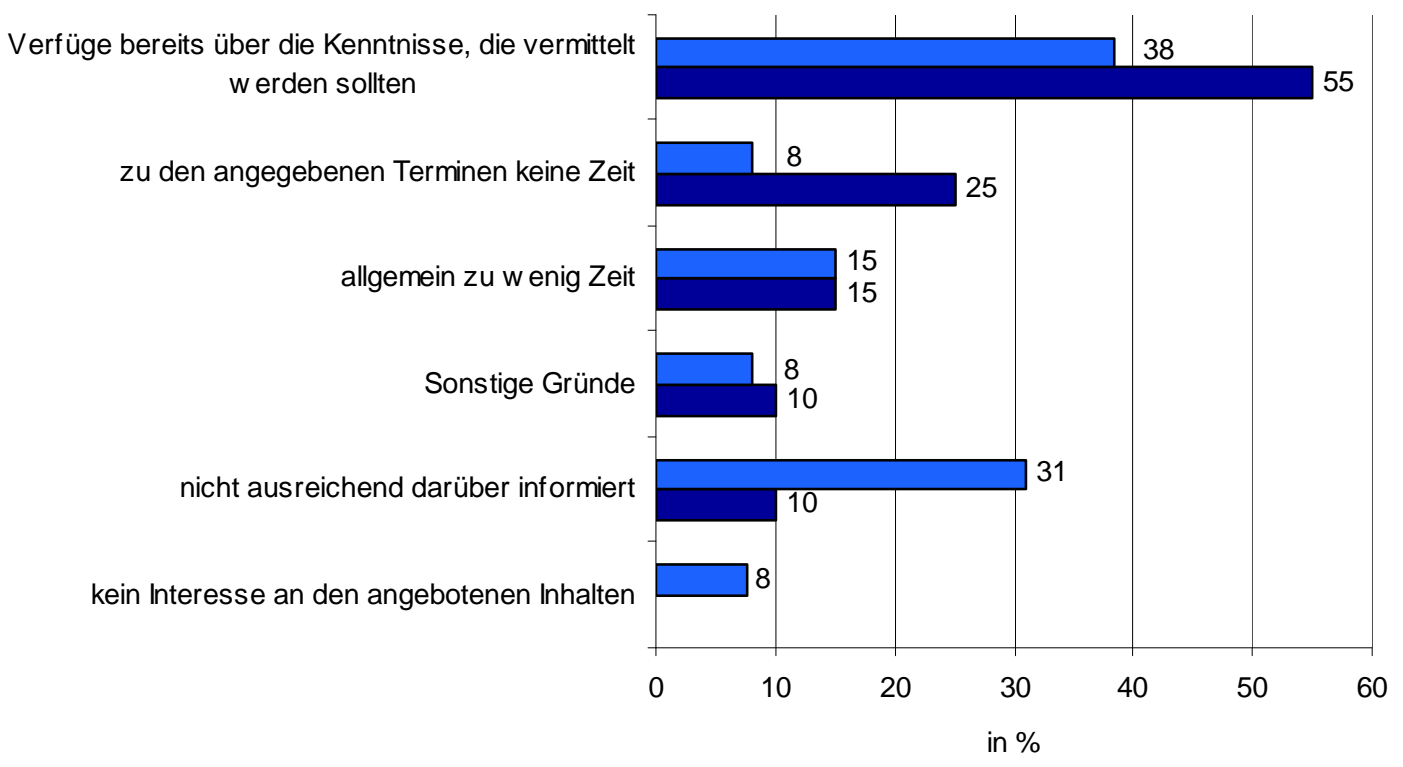

BP-Workshop $\square$ Enreichberatung

Quelle: KMU FORSCHUNG AUSTRIA, n=14 für Einreichberatung bzw. n=23 für BP

Im Oktober 2006 wurde der erste und bislang einzige we-Workshop in Wien durchgeführt, an dem 49 Unternehmer/innen und Gründer/innen aus den Bereichen Mode, Musik, Multimedia und Design teilnahmen. Das von den Calls unabhängig angebotene Ausbildungsangebot „we - Workshop“ wurde jedoch von nur 2 der im Rahmen der gegenständlichen Evaluierung befragten Unternehmen in Anspruch genommen, wobei es jedoch immerhin rd. $30 \%$ der Unternehmen bekannt war. Die Nicht-Teilnahme an den we-Workshops wird dabei von einem Großteil der Unternehmen (56\%) mit dem für kleine und mittlere Betriebe - aufgrund der starken Eingebundenheit in das Alltagsgeschäft - typischen Zeitmangel begründet (vgl. Grafik 7).

Knapp $40 \%$ der befragten Unternehmen gaben an, über die in dem Workshop zu vermittelnden Kenntnisse bereits zu verfügen (wiederum stehen dieser Einschätzung die Angaben der Expert/innen entgegen) und bei immerhin $28 \%$ scheinen die mit $€ 300$,- vergleichsweise hohen Kosten eine prohibitive Wirkung auf die Teilnahme zu haben. Dies könnte unter anderem auch ein Grund dafür sein, dass der für das Jahr 2007 geplante Workshop aus Mangel an Anmeldungen abgesagt werden musste. ${ }^{4}$ So gehen etwa auch Expert/innen davon aus, dass ein niederschwelligeres Weiterbildungsangebot eine verstärkte Teilnahme begünstigen würde.

Ein weiterer Grund könnte gemäß der geführten Interviews auch darin liegen, dass der Workshop angesichts der in der Kreativwirtschaft vergleichsweise geringen Zahl an Gründungen mit hohem Wachstumspotenzial zu schnell wieder angeboten wurde (nur 6 Monaten nach dem ersten we-Workshop). 
Grafik 7 Gründe der Nicht-Teilnahme am we-Workshop, Betriebe in \%

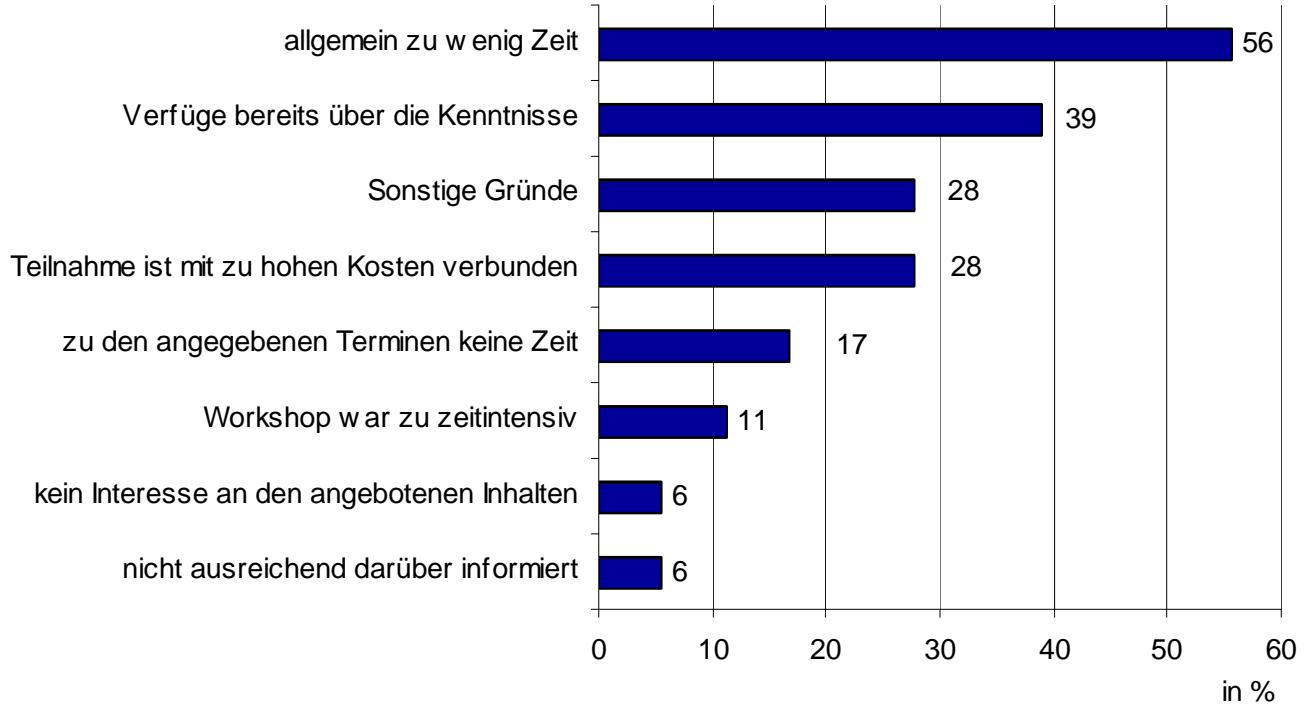

Quelle: KMU FORSCHUNG AUSTRIA, Mehrfachnennungen möglich, $n=34$

Im Rahmen der gegenständlichen Evaluierung wurde auch nach dem Bedarf an Weiterbildung bei den einreichenden und geförderten Unternehmen von iP gefragt. Insbesondere drei Bereiche stechen dabei hervor: rechtliche Fragestellungen, das Thema Finanzierung und der Bereich Kooperationen. Ein Bedarf nach Weiterbildung in rechtlichen Fragestellungen wurde von rd. $37 \%$ der befragten Unternehmen artikuliert (vgl. Grafik 8). Dieser Bedarf ist vor allem bei Mikrounternehmen (bis zu 10 Mitarbeiter/innen) besonders stark und wird vor allem in der Zukunft noch an Bedeutung gewinnen. Die Begründing hierfür liegt in den geforderten Inhalten, die sich in erster Linie am Themenkomplex „Schutz und Verwertung des geistigen Eigentums“ (Intellectual Property Rights, IPR) orientieren, welcher auch eine thematische Klammer für die drei genannten wichtigsten Weiterbildungsbereiche bildet:

„Begleitmaßnahmen im Bereich IPR und allgemein Rechte wären sinnvoll. Kooperationsverträge sind besonders in der Kreativwirtschaft ein wichtiges Instrument um Konfliktpotenzial frühzeitig zu entschärfen." (Förderempfänger 2. Call).

Die steigende Bedeutung von Methoden und Instrumenten zum Schutz und zur Verwertung von geistigem Eigentum ist Gegenstand zahlreicher Untersuchungen ${ }^{5}$, wobei die Befunde darauf schließen lassen, dass Schutzmechanismen wie Patente, Marken, Geschmacksmuster aber auch informelle Schutzmethoden wie das bewusste Management von Geschäftsgeheimnissen einen erheblichen Nutzen unter anderem für die Durchführung von Kooperationen (da dann die Verwertung des geistigen Eigentums klar geregelt ist und somit ein Vertrauensverhältnis zwischen den Partnern geschaffen werden kann) bzw. für die Eigenvermarktung stiften können. Speziell junge Unternehmen haben über das Vehikel IPR bei Verhandlungen mit Kapitalgebern eine

vgl. hierzu z.B. Radauer et al. (2007): Benchmarking National and Regional Support Services for SMEs in the Field of Intellectual Property Right (IPR). Brussels: European Commission, DG Enterprise. 
Möglichkeit, bislang fehlende Kundenreferenzen durch z.B. Patente zu substituieren und damit den erfolgversprechenden Charakter ihrer betrieblichen Aktivitäten zu unterstreichen.

In vielerlei Hinsicht dürfte die Diskussion um das Thema IPR auch den Kern der betrieblichen Aktivitäten, das Geschäftsmodell der Unternehmen, berühren: Fragen, wie etwa wesentliche Einkommensströme heutzutage generiert werden (z.B. über Lizenzvergaben an Fremdfirmen) oder Entscheidungen ob Teile des Produktes gratis oder kostenreduziert angeboten werden sollen, während andere Teile oder Produktdienstleistungen diese Gratisangebote quersubventionieren, haben hinsichtlich ihrer praktischen Durchsetzbarkeit oftmals unmittelbaren IPR Bezug. Die Kreativwirtschaft ist von diesem Thema angesichts der Tatsache, dass ihre Leistungen im digitalen Zeitalter meist leicht und kostengünstig kopierbar sind - man denke hier etwa an die laufende Diskussion um Musiktauschbörsen im Internet - besonders betroffen. Ohne dieses komplexe Themenfeld weiter auszuführen, soll an dieser Stelle vor allem festgehalten werden, dass ein etwaiges IPR Workshopprogramm im Rahmen von iP Cl spezifische Elemente, den Konnex zu verschiedenen Geschäftsmodellen und das Management unterschiedlicher sich ergänzender Schutzrechtsinstrumente berücksichtigen sollte, sprich, es bedarf eines ganzheitlichen Ansatzes der das Themenfeld "Management von geistigen Eigentumschutzrechten" auf betriebswirtschaftlicher Sicht behandelt. ${ }^{6}$

Der Bereich „Kooperationen“, in dem etwas mehr als ein Drittel der befragten Unternehmen Weiterbildungsbedarf sieht, kann natürlich nicht nur durch die Behandlung von Fragen des geistigen Eigentums erarbeitet werden. Hier müssen vielmehr auch andere Aspekte, wie etwa das Management derartiger betrieblicher Kollaborationsaktivitäten, berücksichtigt werden. Vielfach dürfte der Bedarf an Weiterbildung in diesem Bereich aber auch mit dem Wunsch nach einer stärkeren Instrumentalisierung der Vernetzungsbemühungen in den $\mathrm{Cl}$ mit Hilfe des ImpulsProgramm creativwirtschaft gekoppelt sein, wie sich aus einigen vorgebrachten Verbesserungsvorschlägen ableiten lässt:

„...[eine] Vernetzung der Teilnehmer bzw. der geförderten Unternehmen wäre sinnvoll. Ziel wäre [der] Erfahrungsaustausch zu Fördererfahrungen, aber auch sonstigen Themen..." (Einreicher)

„...Verbesserungsmöglichkeiten: Unterstützung in der Vermarktungsstrategie in Form von Kontakten, Netzwerken..." (Förderempfänger 1. Call)

Der Bedarf an zusätzlichen Kooperations- und Vernetzungsaktivitäten ist auch im Zusammenhang mit der kleingliedrigen Struktur der $\mathrm{Cl}$ in Österreich und der oftmals notwendigen Erreichung kritischer Massen im internationalen Kontext zu sehen - viele Bereiche der $\mathrm{Cl}$ sind, gemäß Expert/innen, auf internationaler Ebene mit einem erheblichen strukturellen Wandel konfrontiert, speziell in den IT-nahen Bereichen der CI.

Für weitere Ausführungen sei auf Radauer et al. (2007): Benchmarking National and Regional Support Services for SMEs in the Field of Intellectual Property Right s(IPR). Brussels: European Commission, DG Enterprise verwiesen. 
Als Beispiel hierfür kann die Entwicklung von Computerspielen herangezogen werden: Wurden vor zehn bis zwanzig Jahren Computerspiele noch von relativ kleinen Teams von Programmierer/innen entwickelt (oftmals auch nur von einzelnen Programmierer/innen), so ist es heutzutage nicht unüblich, dass an der Erstellung eines Computerspiels mehr als 100 Personen (Schauspieler, Grafiker, Musiker, Coder, Tester etc.) mitwirken. ${ }^{7}$

Im Lichte dessen ist auch ein weiterer von Expert/innen wie Förderwerbern genannter Ansatzpunkt für Verbesserungen im Programm evident geworden (der aber unmittelbar keinen Bezug zu Weiterbildungsmaßnahmen hat): Hilfestellung bei den notwendigen Internationalisierungsbemühungen der Unternehmen in den $\mathrm{Cl}$, wie beispielsweise von folgendem Einreicher dargelegt wird:

„Kreativität ist keine regionale oder nationale Dienstleistung mehr. Durch die internationale Globalisierung ist sehr starke internationale Konkurrenz vorhanden. Veranstaltungen sollten deshalb auch auf diese Problematik eingehen bzw. Teilnehmer dahingehend unterstützen." (Einreicher)

Hierzu ist jedoch anzumerken, dass das derzeitige Instrumentarium von iP nicht geeignet ist, die geforderten Vernetzungsbemühungen oder die Internationalisierungstendenzen auf breiter Basis zu fördern - angesichts des wohl notwendigen Ressourcenausbaus ist die Zweckmäßigkeit der Erweiterung des ImpulsProgramm um derartige Angebote (z.B. Vernetzungsveranstaltungen, Auftritte auf internationalen Messen u.ä.) jedoch zu prüfen - auch vor dem Hintergrund einer möglichen Zielüberfrachtung bzw. kann dieser Themenbereich als zukünftige Herausforderung für das Förderprogramm gesehen werden.

Abschließend überrascht die geringe geäußerte Nachfrage nach Workshops mit betriebswirtschaftlichen Inhalten, speziell nach solchen die sich mit der Erstellung von Businessplänen befassen (nur $16 \%$ der befragten Betriebe würden sich hier zusätzliche Angebote wünschen); wie bereits angesprochen ist dieser Sachverhalt vorsichtig zu interpretieren, denn viele Unternehmer/innen.

Dem ist allerdings auch die These gegenüberzustellen, dass den Businessplänen möglicherweise bislang eine zu zentrale Rolle im Förderprogramm eingeräumt wurde und diese Rolle in der Förderlogik differenzierter, nach dem Entwicklungstand des Unternehmens bzw. auch der Projektideen, betrachtet werden sollte - seit kurzem wird z. B. bei länger bestehenden Unternehmen die Vorlage eines Businessplans nicht mehr verlangt, was im Sinne der Zweckmäßigkeit (länger existierende Betriebe haben die Praktikabilität ihres Businessmodells auch mit der andauernden Existenz des Betriebs unter Beweis gestellt) und einer Reduktion des administrativen Aufwandes auch vom Team der Evaluator/innen unterstützt wird. Bei jungen Unternehmen bzw. Unternehmen in Gründung erscheint es in der Mehrzahl hingegen durchaus sinnvoll, einen Businessplan zu verlangen. In vielen Fällen befinden sich interessante Geschäfts- und Projektideen aber auch in einem derart frühen Zustand, dass die Erstellung eines komplexen Businessplans als nicht zielführend bezeichnet werden kann und damit auch eine Einreichung bei den iP Calls wenig erfolgversprechend ist.

\footnotetext{
Siehe hierzu auch die Ausführungen von WIKIPEDIA (http://de.wikipedia.org/wiki/Computerspiel\#Entwicklerszene) bzw. Wardell, Brad (2006): Postmortem: Stardock's Galactic Civilizations II: Dread Lords (http://www.gamasutra.com/features/20060405/wardell_01.shtml )
} 


\section{Grafik 8 Bereiche mit Weiterbildungsbedarf, Betriebe in \%}

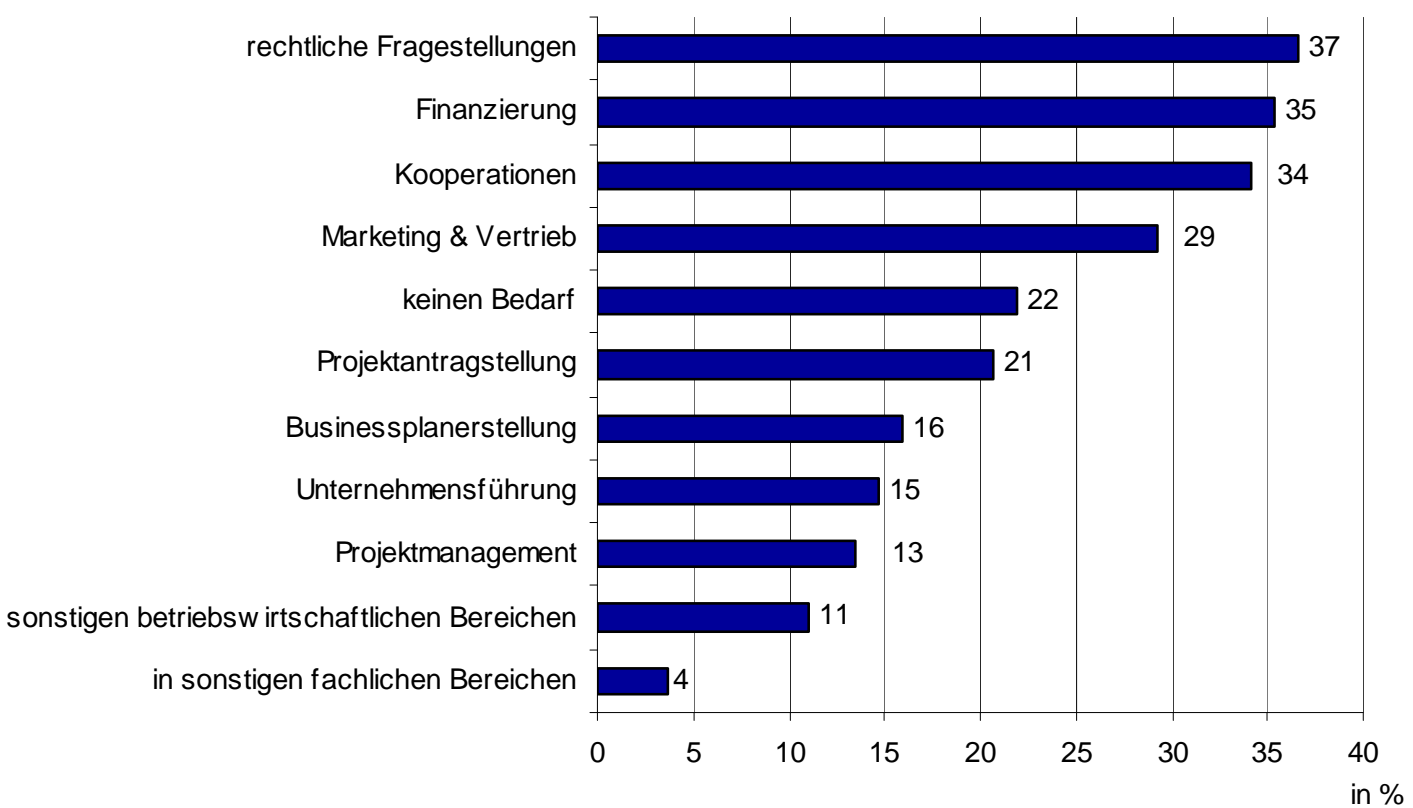

Quelle: KMU FORSCHUNG AUSTRIA, Mehrfachnennungen möglich, n=82

Hier wäre die Etablierung eines eigenen Segments im ImpulsProgramm creativwirtschaft anzudenken (eine mögliche „iP Light“-Schiene) um derartige Protoprojekte anzuschieben. Bei einer derartigen „iP Light“ Förderung müsste aber auch berücksichtigt werden, den Threshold für die Inanspruchnahme der Förderung nicht zu niedrig zu gestalten. Andernfalls könnte eine erhebliche Zahl der dann einlangenden Anträge hohe Kosten bzw. einen hohen administrativen Aufwand verursachen, der den Nutzen der Programmschiene konterkarieren würde. 


\section{$5 \quad$ Analyse der Mobilisierungswirkungen des Programms}

Zielgruppe des ImpulsProgramm creativwirtschaft sind Unternehmen aus den Bereichen Multimedia, Design und Musik der österreichischen Kreativwirtschaft. Neben den Herausforderungen die sich aufgrund der sehr kleingliedrigen Struktur der Kreativwirtschaft und der in diesem Bereich agierenden, eher förderfernen Akteure in Hinblick auf die Effektivität eines Förderprogramms in diesem Wirtschaftssegment ergeben, stellt das ImpulsProgramm creativwirtschaft zudem auch recht hohe Ansprüche an den innovativen Gehalt und die Vermarktungskompetenz der zu fördernden Projekte und Akteure (es werden nur Projekte gefördert, die nicht nur eine hohe Relevanz für den jeweiligen Kreativbereich haben sondern auch eine erfolgreiche wirtschaftliche Umsetzung erwarten lassen bzw. einen Wertschöpfungsprozess generieren). Bevor die Evaluierung daher auf die Wirkungen des Programms auf der Projektebene eingeht, werden im Folgenden zunächst die Mobilisierungswirkungen von iP analysiert, d.h. die Erreichung der Zielgruppe und damit auch die Breitenwirkung des Programms.

\subsection{Zielgruppenereichung}

Die breite Öffentlichkeitsarbeit insbesondere im Rahmen der Einführung des ImpulsProgramm creativwirtschaft brachte ein vergleichsweise hohes Echo. Bei der ersten Ausschreibung im Jahr 2004/2005 wurden insgesamt 315 Projekte eingereicht (wovon 24 gefördert wurden). Seit dem 1. Call hat sich die Anzahl der Einreichungen jedoch stetig reduziert. Im 2. Call im Jahr 2005/2006 wurden nur noch 159 (davon 16 geförderte) und im 3. Call, der 2007 erfolgte, nur noch 123 Projekte eingereicht (siehe Tabelle 2). Die große Anzahl der Einreichungen im 1. Call lässt sich u. a. damit erklären, dass sich bei der erste Ausschreibung viele Personen/Unternehmen angesprochen fühlten, die jedoch nicht explizit der Zielgruppe des Programms zugeordnet werden konnten. Durch die Adjustierung der Ausschreibungsunterlagen und anhand sichtbarer Beispiele von geförderten Projekten wurden die Zielgruppe und damit auch die förderbaren Tatbestände/Projektinhalte nach und nach präzisiert, wodurch sich die Anzahl der Einreichungen reduziert hat. Diese Beobachtung wird auch von der Entwicklung der Erfolgsquoten im Rahmen der ersten beiden Calls gestützt. Betrug diese im 1. Call mit 315 eingereichten und 24 geförderten Projekten noch $7 \%$ so ist diese im 2. Call mit einem Verhältnis von 159/16 zwischen eingereichten und geförderten Projekten auf immerhin 10\% gestiegen. Nicht zuletzt wird die gestiegene Qualität der Projektanträge auch von den im Zuge der Evaluierung interviewten Juroren/innen hervorgestrichen. Es könnte des Weiteren könnte aber auch eine gewisse Masse an Projektideen im $\mathrm{Cl}$ Bereich bestehen, die sich in Ermangelung ähnlich gelagerter Förderprogramme gebildet hat - mit den laufenden Erfahrungen zur Förderpraxis durch iP würden viele dieser Ideenträger nunmehr von einer Einreichung absehen (eben weil die Ideen noch nicht weit genug gediegen sind für die iP Förderung), was dem Argument einer iP Light Schiene (siehe Abschnitt 4.3) weiteren Vorschub geben würde.

Insgesamt wurden im Rahmen des ImpulsProgramm creativwirtschaft bisher 53 Projekte im Rahmen von drei Calls gefördert, $€ 4,7$ Mio an Fördermitteln vergeben und damit ein Projektvolumen von insgesamt $€ 12,6$ Mio unterstützt. $€ 2,1$ Mio der vergebenen Fördermittel entfielen dabei auf den 1. Call und jeweils rd. $€ 1,3$ Mio auf den 2. und 3. Call. Damit wird das ImpulsProgramm creativwirtschaft, den interviewten Experten/innen zu Folge, im Bereich der Förderung von Unternehmen aus der Kreativwirtschaft sowohl hinsichtlich der Höhe der Fördersummen als auch der Vergabepraxis im internationalen Feld als gutes Beispiel angesehen. 
Tabelle 2 Anzahl und Projektkosten der geförderten und eingereichten Projekte

\begin{tabular}{l|l|c|c|c|c}
\hline & & Musik & Multimedia & Design & GESAMT \\
\hline $\begin{array}{l}\text { Anzahl der } \\
\text { eingereichten/ } \\
\text { geförderten } \\
\text { Projekte }\end{array}$ & 1.Call & $83 / 5$ & $123 / 11$ & $109 / 8$ & $315 / 24$ \\
\cline { 2 - 6 } & 2. Call & $37 / 5$ & $78 / 6$ & $44 / 5$ & $159 / 16$ \\
\cline { 2 - 6 } & 3. Call & $14 / 1$. & $61 / 7$ & $48 / 5$ & $123 / 13$ \\
\hline \multirow{2}{*}{$\begin{array}{l}\text { vergebene } \\
\text { Mio } €\end{array}$} & 1.Call & 0,256 & 1,018 & 0,758 & 2,13 \\
\cline { 2 - 6 } & 2. Call & 0,409 & 0,515 & 0,385 & 1,31 \\
\cline { 2 - 6 } & 3. Call & 0,080 & 0,716 & 0,552 & 1,35 \\
\hline \multirow{2}{*}{$\begin{array}{l}\text { gefördertes } \\
\text { Projektvolumen } € \text { Mio }\end{array}$} & 1.Call & 0,8 & 2,7 & 2,3 & 5,8 \\
\cline { 2 - 6 } & 2. Call & 1,0 & 1,8 & 1,0 & 3,8 \\
\cline { 2 - 6 } & 3. Call & 0,16 & 1,7 & 1,1 & 3,0 \\
\hline
\end{tabular}

Quelle: Daten des ImpulsProgramm creativwirtschaft, eigene Darstellung der KMU FORSCHUNG AUSTRIA

Der wichtigste Förderbereich, dem die meisten Einreichungen und letztlich auch geförderten Projekt zuzuordnen sind, ist der Bereich Multimedia. Der Bereich Design steht an zweiter Stelle. Zwischen dem 1. und den weiteren beiden Calls hat sich dabei in allen Bereichen die Anzahl der Einreichungen reduziert, mit Ausnahme des Bereiches Design, in dem die Anzahl der Einreichungen im 3. Call leicht gestiegen ist. Der Musikbereich weist den stärksten Abwärtstrend in Bezug auf die Anzahl der Einreichungen seit dem 1. Call auf (vgl. Grafik 9). Mit nur 14 eingereichten Anträgen im 3. Call kann hier wohl auch auf erste Sättigungstendenzen geschlossen werden (bzw. kann dies auch als Hinweis gewertet werden, dass das Design der derzeitigen iP Förderung nicht den Gegebenheiten des Musikbereichs entspricht). Die geringe Anzahl sowie auch die oftmals unbefriedigende Qualität der Anträge im Bereich Musik haben denn auch zu der Entscheidung geführt, dass der Musikbereich ab 2008 in der derzeitigen Form nicht mehr Teil der iP Förderung sein wird.

\section{Grafik $9 \quad$ Anzahl der Einreichungen, nach Call und Bereich}

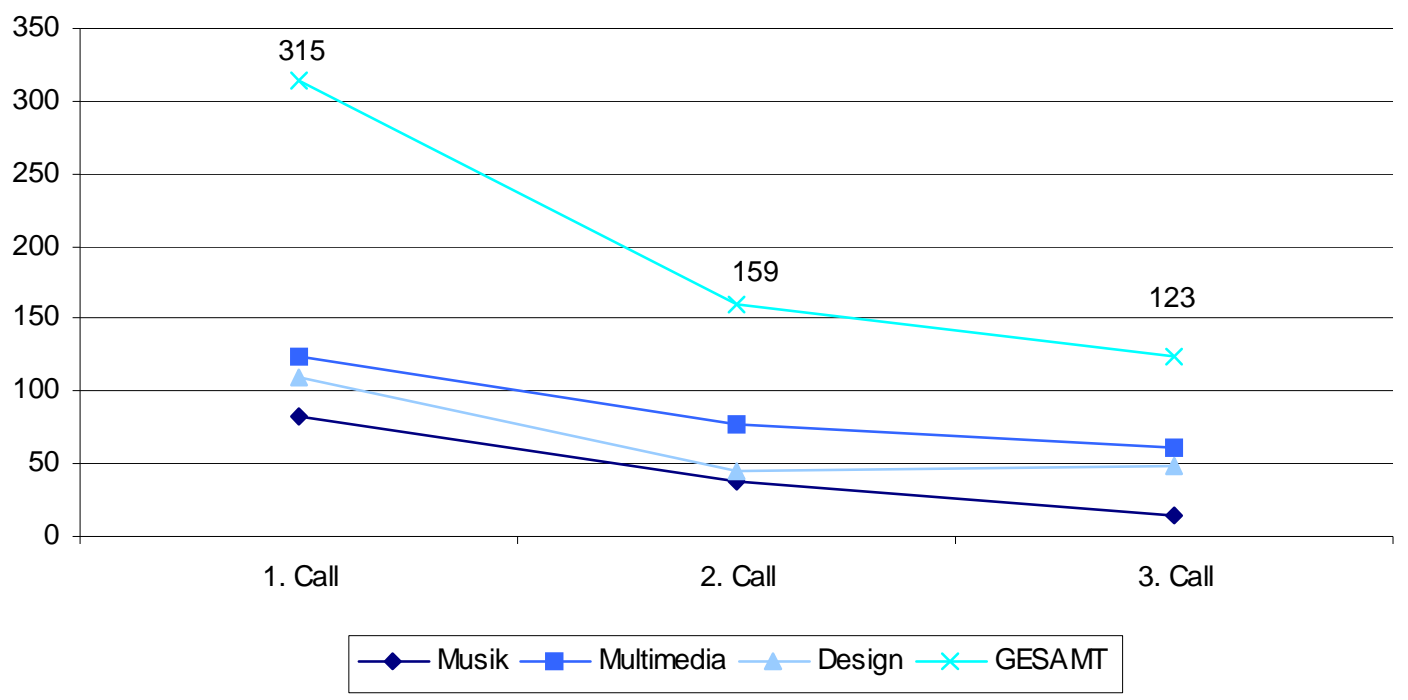

Quelle: ImpulsProgramm creativwirtschaft, eigene Berechnungen, $n=597$ 
Laut den interviewten Expert/innen wurde der Programmleitung ein Konzept vorgelegt, dass eine neue Förderungsschiene für die Musik vorsieht, bei dem es zu einer verstärkten Kooperation mit dem Musikfonds kommen soll. Diese Förderung würde sich der Förderung der Musikverwertung und nicht der Musikproduktion widmen mit einem Schwerpunkt auf der Exportschiene. Projekte, die nicht darunter fallen, wie z. B. Einreichungen durch Instrumentenbauer und Online-Musikplattformen, könnten im Bereich Design bzw. Multimedia eingereicht werden. Fragwürdig erscheint in diesem Zusammenhang jedenfalls, ob sich z.B. ein Musikinstrumentenbauer, der keine besonderen Designleistungen in seine Projekte einfließen lässt und dessen Projekte auch keine multimedialen Charakteristika aufweisen, in den anderen Förderschienen von iP wieder findet. Das neue Konzept für den Musikbereich ist gemäß dem Informationsstand der Evaluator/innen noch nicht approbiert. Der Musikbereich dürfte vor der Herausforderung bestehen, förderbare (auch im Sinne von EURichtlinienkonforme) Tatbestände mit Kreativitäts- und technischem Innovationsgehalt zu definieren, die letztlich auch keine reine Künstlerförderung darstellen.

Der Bereich Musik demonstriert auch eindrucksvoll die Notwendigkeit, in den gem. Expert/innen prinzipiell gut gewählten Bereichen seitens des Förderwerbers individuell vorzugehen und weitere entsprechende Nachjustierungen bei der Definition der Zielgruppen in Abhängigkeit der bisherigen Erfahrungen vorzunehmen. Eine Möglichkeit bestünde in dieser Hinsicht in der bewussten Ansprache der unterschiedlichen Unternehmenstätigkeitsprofile (für die Unternehmenstätigkeitsprofile siehe Grafik 14) in den drei Förderbereichen, speziell hinsichtlich der expliziten Unterscheidung von „Lieferanten“ für die $\mathrm{Cl}$ (hier könnten dann Musikinstrumentenbauer explizite Erwähnung finden oder im Designbereich der Bereich der Halbfabrikate - z.B. ein Hersteller von kunstvollen Fliesen oder Fliesenelementen, die dann von Designern weiterverarbeitet werden) und „reinen CI Unternehmen“ (d.h. beispielsweise Produkte von Designern).

Hinsichtlich der regionalen Verteilung der an iP teilnehmenden Unternehmen dominiert Wien mit einem Anteil von rd. 40 - $45 \%$ der Einreichungen im Rahmen der drei bisher durchgeführten Calls. An zweiter Stelle und mit deutlichem Abstand zu den anderen Bundesländern folgt die Steiermark, wobei sich hier die Anzahl der Einreichungen vom 2. zum 3. Call um nahezu ein Drittel reduziert hat. Salzburg ist das einzige Bundesland, in dem die Anzahl der Einreichungen im Zeitverlauf der drei Calls leicht, aber dennoch stetig gestiegen ist. Entgegen dem Österreich-weiten Trend können darüber hinaus die Bundesländer Niederösterreich und Tirol auf gestiegene Antragszahlen im 3. verglichen mit den ersten beiden Calls verweisen. In Oberösterreich wurde im Jahr 2007 immerhin wieder das Ausgangsniveau des 1. Calls im Jahr 2004/2005 erreicht (vgl. Grafik 10). 


\section{Grafik 10 Regionale Verteilung der Einreichungen, nach Bundesländer und nach} Call, in \%

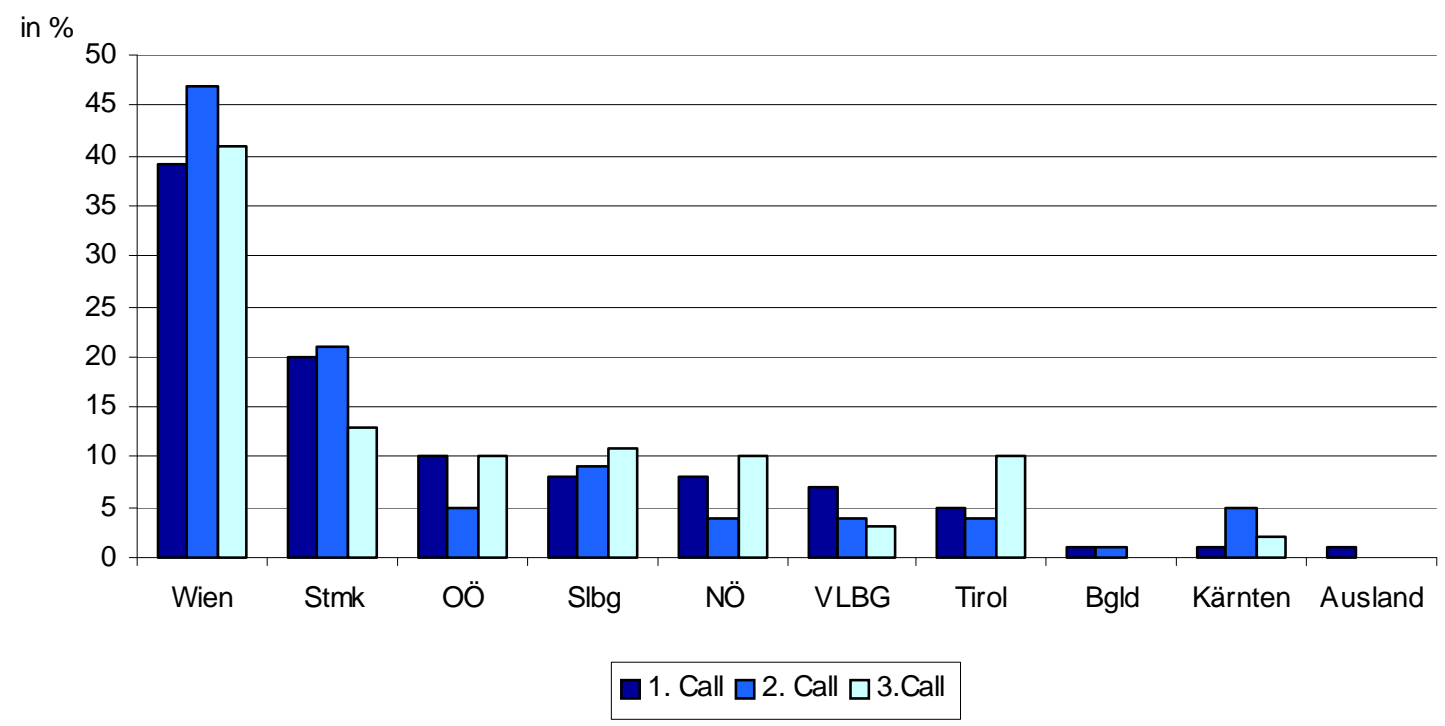

Quelle: KMU FORSCHUNG AUSTRIA, n = 597

Insgesamt ist im Rahmen der Analyse der Zielgruppenerreichung zunächst festzustellen, dass das in der ersten begleitenden Evaluierung erwähnte Spannungsfeld zwischen technisch orientierter Innovationsförderung einerseits und Kreativförderung andererseits im 2. und 3. Call in dieser Form nicht mehr zu beobachten war. Auch die stetig gesunkenen Antragszahlen im Rahmen der drei bisher durchgeführten Calls lassen eher auf eine Präzisierung und stärkere Fokussierung des ImpulsProgramm creativwirtschaft, denn auf Sättigungstendenzen im Feld schließen. Augenscheinlich ist es gelungen, die Richtlinientexte derart umzuformulieren und die Änderungen auch in entsprechender Weise zu kommunizieren, dass sowohl unter den Juror/innen und Expert/innen als auch bei den antragstellenden Unternehmer/innen weitestgehende Klarheit über die förderbaren Tatbestände/Projektinhalte besteht. Zu begrüßen sind in diesem Zusammenhang vor allem auch die verstärkten Kooperationsbemühungen mit Multiplikatoren auf regionaler Ebene, um das Programm in Zukunft wieder aktiver und breiter zu bewerben (aber auch um einer abgestimmten Förderstrategie in Österreich Vorschub zu leisten). Durch den begonnenen Aufbau von Kooperationen mit regionalen Netzwerk- und Technologiepartnern hat das ImpulsProgramm bereits wichtige Arbeit geleistet, die in jedem Fall fortgesetzt werden sollte. 


\subsection{Struktur der bei iP teilnehmenden Unternehmen}

Zielgruppe des ImpulsProgramm creativwirtschaft sind in erster Linie kleine und mittlere Unternehmen (KMU) die in den Bereichen Multimedia, Design und Musik der Kreativwirtschaft aktiv sind. Die kleingliedrige Struktur der österreichischen Kreativwirtschaft spiegelt sich so auch im Sample der geförderten Unternehmen aus dem 1. und 2. Call sowie den einreichenden Unternehmen des 2. und 3. Calls wieder. Ein Fünftel der Unternehmen sind Ein-Personen-Unternehmen (EPU) und haben keine Mitarbeiter/innen. Zwei Drittel der einreichenden Unternehmen haben zwischen 1 bis 10 Mitarbeiter/innen, wobei der Medianwert in dieser Beschäftigtengrößenklasse bei 3 Mitarbeiter/innen pro Unternehmen liegt. Der Anteil an Unternehmen mit 11 bis 50 Beschäftigten liegt bei rd. $13 \%$ (vgl. Grafik 11).

Grafik 11 Anzahl der Mitarbeiter/innen bei den Unternehmen des 1. Calls (Förderempfänger), 2. und 3. Call (Förderempfänger und Einreicher), Betriebe in \%

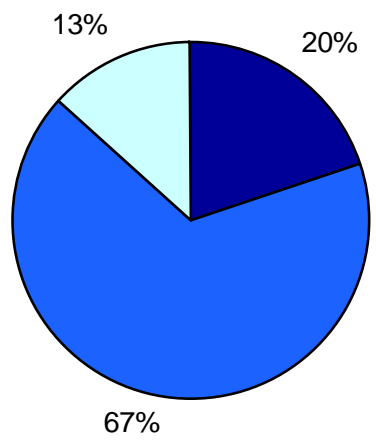

$\square$ EPU $\square 1$ bis 10 MA $\square 11$ bis 50 MA

Quelle: KMU FORSCHUNG AUSTRIA, $n=90$

Rd. ein Drittel der befragten Unternehmen hat im Jahr 2006 einen Umsatz von weniger als $€ 50.000$ erwirtschaftet. Bei einem Viertel der Unternehmen lag der Umsatz 2006 zwischen $€ 50.001$ und $€ 100.000$ und bei rd. $30 \%$ zwischen $€ 100.001$ und $€ 500.000$. Nur $10 \%$ der befragten Unternehmen konnten 2006 einen Umsatz von mehr als $€ 500.000$ lukrieren (vgl. Grafik 12). 
Grafik 12 Umsatz im Jahr 2006 der einreichenden Unternehmen des 1., 2. und 3. Calls, Betriebe in \%

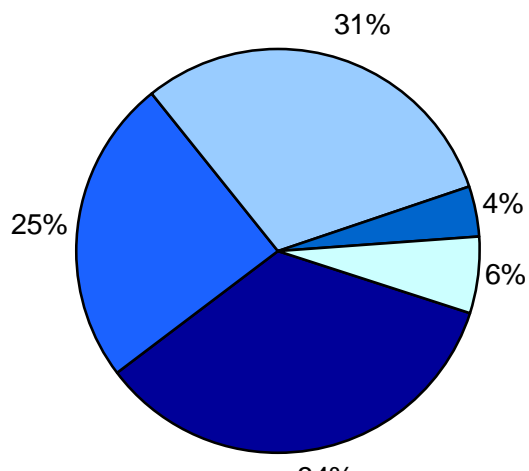

$34 \%$

a bis $€ 50.000 \quad \square € 50.001$ bis $€ 100.000 \quad \square € 100.001$ bis $€ 500.000$

$\square € 500.001$ bis $€ 1.000 .000 \square$ mehr als $€ 1.000 .001$

Quelle: KMU FORSCHUNG AUSTRIA, $\mathrm{n}=81$

Die Vollzeitanstellung ist bei den einreichenden Unternehmen mit $\mathrm{rd} .60 \%$ die meist verbreiterte Form der Beschäftigung, gefolgt von der selbstständigen Beschäftigung mit rd. $47 \%$, der Teilzeitbeschäftigung mit rd. $30 \%$ und der geringfügigen Beschäftigung mit rd. $21 \%$ (vgl. Grafik 13). Unterschiede in der Art der Beschäftigung sind dabei insbesondere zwischen verschiedenen Beschäftigtengrößenklassen festzustellen. Alle einreichenden Unternehmen mit 11 bis 50 Mitarbeiter/innen haben (zumindest eine/n) Vollzeitbeschäftigte/n, wobei dies nur auf $70 \%$ der Unternehmen mit 1 bis 10 Mitarbeiter/innen zutrifft. Dagegen ist die Form der Teilzeitbeschäftigung sowie jene der geringfügigen Beschäftigung bei den Unternehmen von 1 bis 10 Mitarbeiter/innen stärker vertreten.

\section{Grafik 13 Formen der Beschäftigung bei den einreichenden Unternehmen des} 1., 2. und 3. Calls, Betriebe in \%, Mehrfachnennungen möglich

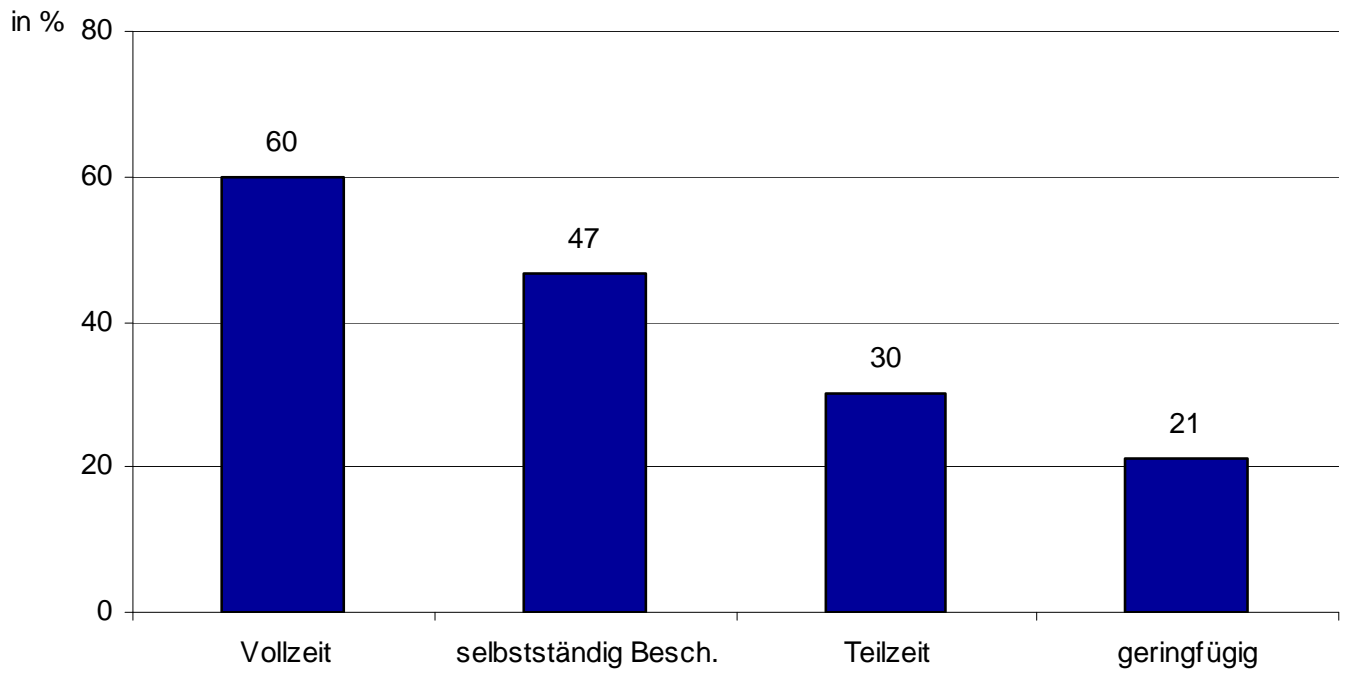


Bei dem Impulsprogramm creativwirtschaft handelt es sich um ein Programm, das sich gezielt an die Bereiche Multimedia, Design und Musik richtet, aber im hohen Maße nach dem bottom-up Prinzip angelegt ist, in dem die Bedürfnisse der Zielgruppen im Vordergrund stehen und nicht die Steuerung durch den Fördergeber. Dieser Tatbestand spiegelt sich auch in der vergleichsweise heterogenen Struktur der teilnehmenden Betriebe wieder, die auch auf den Querschnittscharakter der $\mathrm{Cl}$ hinweist. So weisen die beim 2. und 3. Call einreichenden Unternehmen einen interessanten Mix an Unternehmenstätigkeiten auf. Mehr als die Hälfte der Unternehmen, beschreibt ihre Unternehmenstätigkeit mit der „Erbringung von Dienstleistungen, die (auch) kreativ-künstlerische Elemente beinhalten“. Rd. $42 \%$ der Unternehmen geben an rein kreativ-künstlerische Tätigkeiten zu erbringen und rd. $24 \%$ der unternehmen beschreiben sich als Zulieferer von Dienstleistungen für kreativ-künstlerische Personen/Unternehmen. Es finden sich auch Unternehmen, die der Sachgüterproduktion (eher „traditionellere“ Branchen) zuzuordnen sind, aber Sachgüter mit kreativkünstlerisch Elemente (rd. ein Fünftel der Unternehmen) bzw. für kreativ-künstlerisch tätige Personen/Unternehmen ( $r d .13 \%$ der Unternehmen) produzieren (vgl. Grafik 14).

Grafik $14 \quad$ Unternehmenstätigkeit der einreichenden Unternehmen des 2. und 3. Calls, Betriebe in \%

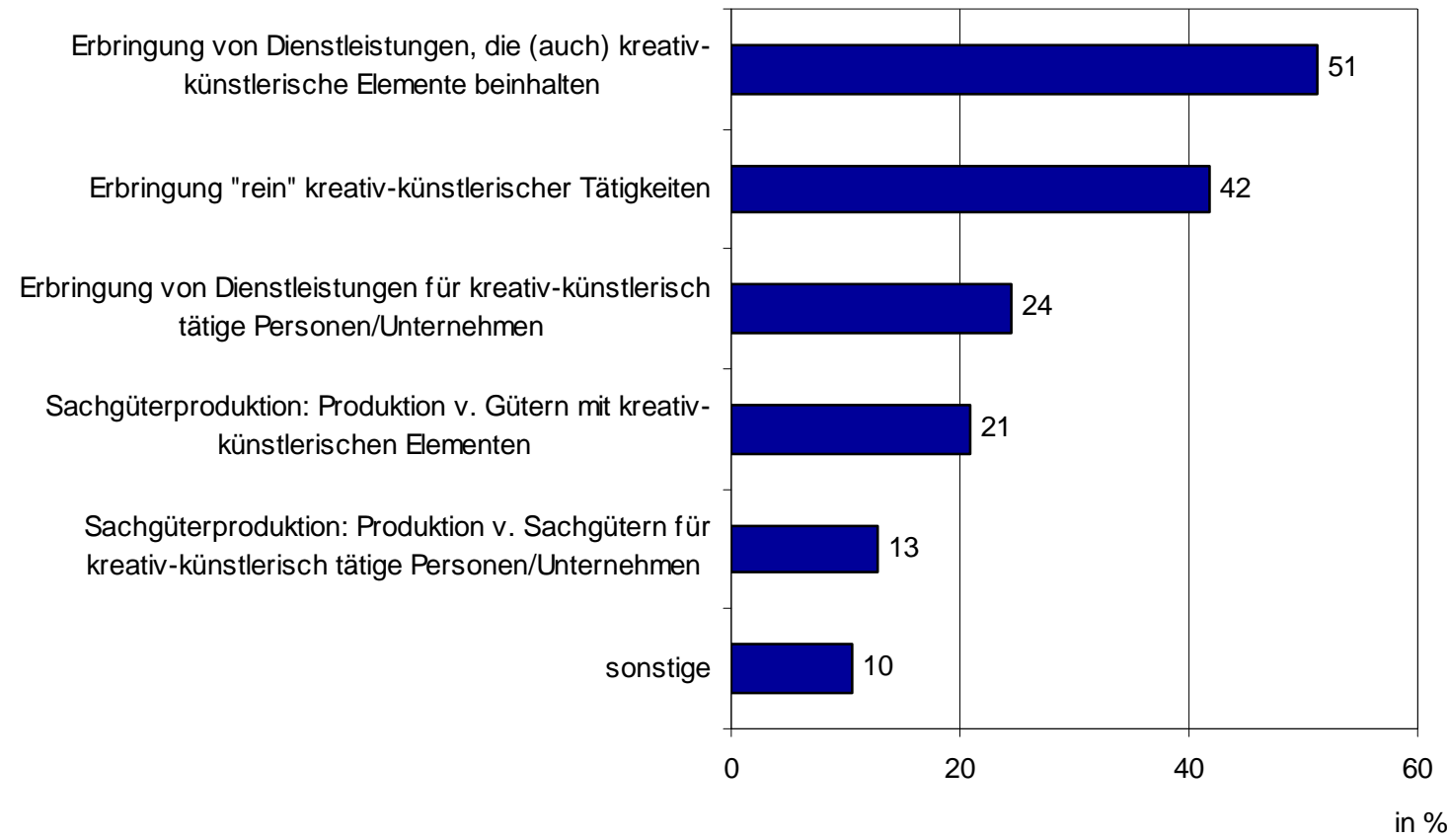

Quelle: KMU FORSCHUNG AUSTRIA, Mehrfachnennungen möglich, $n=86$ 
Der Anteil der Unternehmen, die in den letzten fünf Jahren Preise bzw. Stipendien erhalten haben, ist seit der ersten Ausschreibung kontinuierlich gestiegen. Betrug dieser Anteil im 1. Call nur $3 \%$ und im 2. Call immerhin $6 \%$, so hat er sich im 3. Call auf immerhin ein Fünftel erhöht (vgl. Grafik 15). Bei den erhaltenen Preisen handelt es sich u. a. um regionale Innovationspreise, Nominierungen für den Multimedia Staatspreis, den Adolf-Loos Staatspreis etc. Auch bezüglich der erhaltenen Förderungen ist ein deutlicher Anstieg vom 1. bis zum 3. Call zu beobachten, wenngleich es hier einen deutlichen Einbruch im 2. Call gab. Bei den durch die einreichenden Unternehmen in Anspruch genommenen Förderungen handelt es sich um folgende Programme: FFG (Basisprogramme und thematische Programme), departure, aws / iP, ZIT auf Bundesebene und TICnet, ADA, CAST \& INiTS, WWFF auf Landesebene. Der deutliche Anstieg des Anteils von Preisträgern und Förderempfängern untern den einreichenden Unternehmen kann als weiterer Indikator für die gestiegene Qualität der Anträge bzw. an iP beteiligten Akteure gewertet werden (siehe auch Kapitel 5.1).

\section{Grafik 15 Teilnahme an Förderprogrammen und/oder Beziehen von Preisen/ Stipendien in den letzten 5 Jahren, Betriebe in \%}

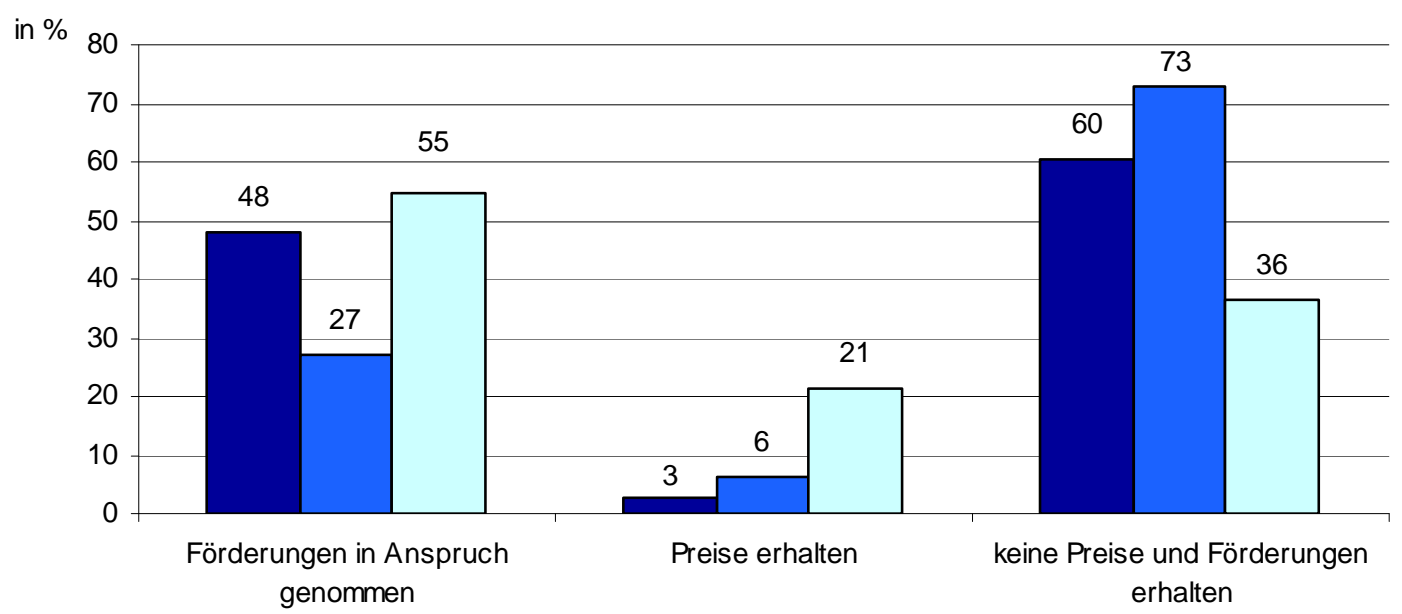

1. Call $\square$ 2. Call $\square$ 3. Call

Quelle: KMU FORSCHUNG AUSTRIA, Mehrfachnennungen möglich, 1.Call (aus der 1. Zwischenevaluierung): $n=73$, 2.Call: $n=33$, 3.Call: $n=33$

Wurden im 1. Call noch rd. $28 \%$ der antragstellenden Unternehmen anlässlich der Teilnahme an iP gegründet, so sank der Anteil derartiger Anlassgründungen im 2. und 3. Call auf jeweils rd. $9 \% .^{8}$ Das ImpulsProgramm creativwirtschaft grenzt sich damit deutlicher als zuvor von Gründungsförderungsprogrammen ab und fokussiert stärker auf bereits etablierte Unternehmen. Die an iP teilnehmenden Unternehmen sind jedoch dennoch sehr jung: die Hälfte der einreichenden Unternehmen des 2. und 3. Calls wurde nach 2003 gegründet. Auffallend ist auch der niedrige Frauenanteil in Bezug auf die Projektleitung der bei iP eingereichten Projekte: Hinter $87 \%$ der eingereichten Projekte stehen Männer als (potenzielle) Projektleiter, nur für $13 \%$ der eingereichten Projekte zeichnet eine Frau verantwortlich. Die im Erfolgsfall von einer Frau geleiteten Projekte sind dabei zu gleichen Teilen in den Bereichen Multimedia, Musik und Design vertreten.

Vier Unternehmen, die für das im Rahmen des ersten Calls eingereichte Projekt neu gegründet wurden, haben im 2. und/oder 3. Call nochmals eingereicht. 


\subsection{Zielgruppenansprache}

Aufgrund der Schwierigkeiten, vor denen die meist sehr kleinen Unternehmen aus dem Kreativwirtschaftsbereich in der Beantragung und Durchführung von Förderprojekten stehen, wurde im Zuge der Öffentlichkeitsarbeit von iP auf unterschiedliche Kanäle zurückgegriffen (z.B. Internetpräsenz mit eigener Domain, die Organisation von Veranstaltungen, Publikation von Foldern, Nutzung von Multiplikatoren und deren Datenbeständen (Bsp: arge creativwirtschaft der Wirtschaftskammer Österreich). Insbesondere im Rahmen des 1. Calls lag einer der Schwerpunkte der Programmaktivitäten auf der Öffentlichkeitsarbeit, um das Programm in der entsprechenden Community gut zu positionieren. Generell gehen die meisten interviewten Expert/innen denn auch davon aus, dass die Bekanntheit von iP in den vergangenen Jahren leicht gestiegen ist. Auch immerhin rd. die Hälfte der an iP beteiligten Unternehmen schätzt das Programm als "sehr bekannt" bzw. „eher bekannt" in der Community ein (siehe Grafik 16). Im Verlauf der 3. Calls hat sich dieser Anteil zwar zum Teil deutlich reduziert, dies dürfte jedoch unter anderem auch auf die erfolgte Fokussierung der Zielgruppe bzw. des Fördergegenstandes zurückzuführen sein (siehe Abschnitt 5.1). Die Tatsache, dass jedoch ein nicht unbeträchtlicher Teil der bei iP einreichenden Unternehmen das ImpulsProgramm creativwirtschaft als eher unbekannt einstuft, dürfte die Einschätzung von Expert/innen bestätigen, wonach durchaus noch Potenzial für eine weitere Diffundierung der Marke „iP“ in der Zielgruppe besteht.

\section{Grafik 16 Einschätzung der Bekanntheit von iP durch einreichende Betriebe, Betriebe in \%}

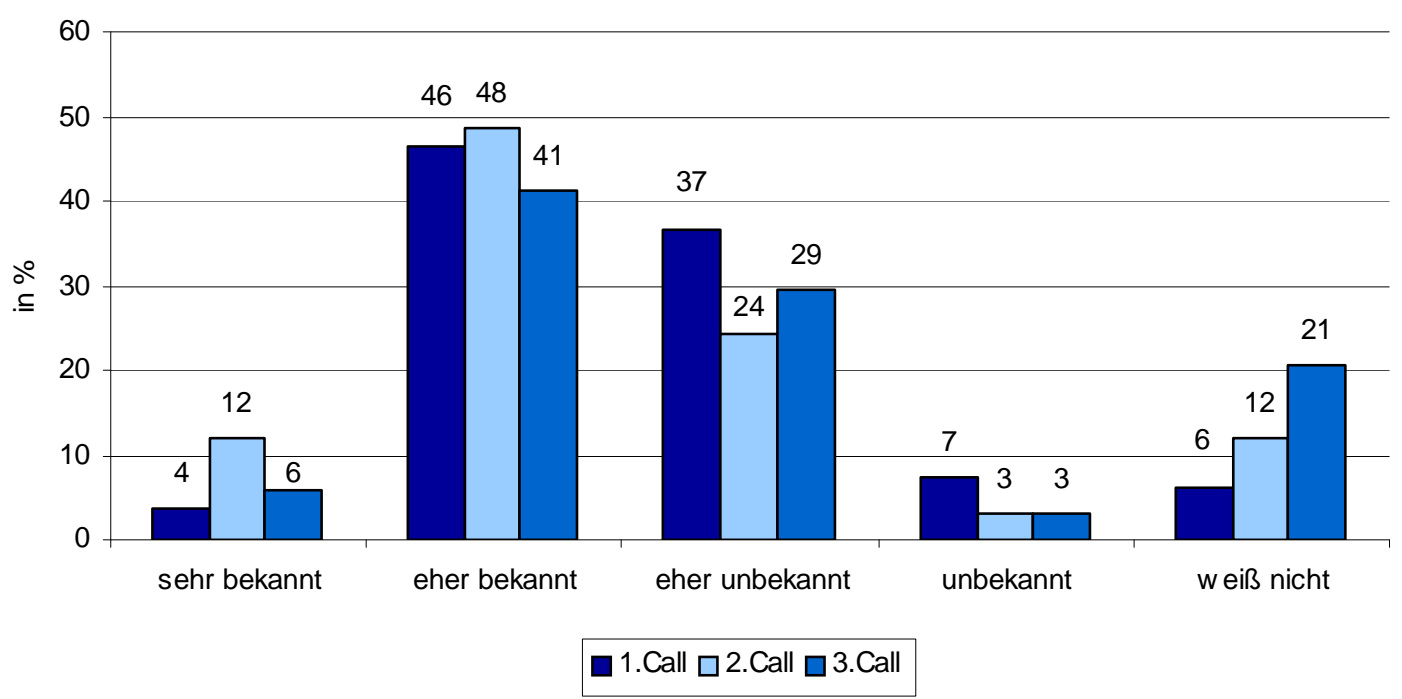

Quelle: KMU FORSCHUNG AUSTRIA, 1.Call: $n=82$ (aus erster Evaluierung), 2.Call: $n=33$, 3.Call: $n=34$

Noch weniger bekannt sind die Leitprojekte von iP. Sie werden auch kaum mit dem ImpulsProgramm bzw. der aws in Verbindung gebracht (vgl. Grafik 17). Die einzige Ausnahme stellt dabei das designforum in Wien dar: Fast zwei Drittel der befragten Unternehmen kennen diese Institution und über $20 \%$ bringen diese mit iP in Verbindung. Das Kreative Handwerk Tirol, das Kreative Handwerk Salzburg und das Austrian Music Ambassador Network (AMAN) kennen weniger als ein Fünftel der befragten Unternehmen. Anzumerken ist jedoch, dass in den Bundesländern, in denen die Leitprojekte implementiert sind, diese über einen höheren Bekanntheitsgrad verfügen, z. B. ist bei zwei Drittel der befragten Salzburger Unternehmen das Kreative 
Handwerk Salzburg bekannt und in Tirol kennt die Hälfte der befragten Unternehmen das Kreative Handwerk Tirol.

\section{Grafik 17 Bekanntheitsgrad der Leitprojekte und deren Bezug zu iP, einreichende} Betriebe des 1., 2. und 3. Calls in \%

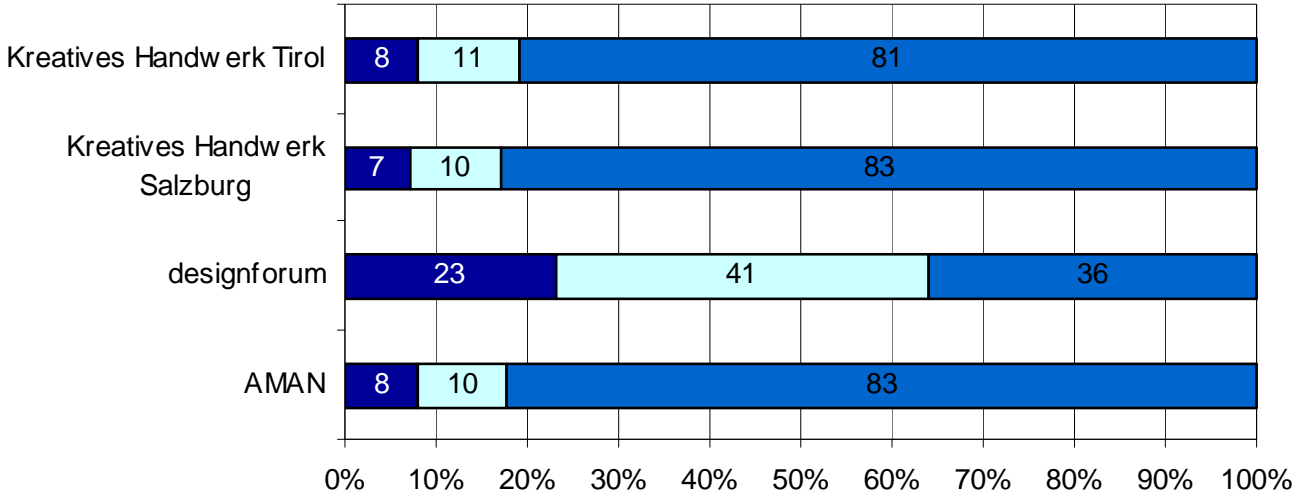

bekannt, als Teil vom ImpulsProgramm/aw s

$\square$ bekannt, aber der Bezug zum Impuls Programmaw s ist mir nicht bekannt

$\square$ nicht bekannt

Quelle: KMU FORSCHUNG AUSTRIA, $n=80$

Die wichtigsten „Vertriebsschienen“ des ImpulsProgramm creativwirtschaft stellen persönliche Kontakte und das Internet dar (vgl. Grafik 18). Zwischen dem 1. und dem 3. Call haben sich dabei kaum Änderungen in Bezug auf die von den einreichenden Unternehmen genutzten Informationskanälen ergeben, lediglich die Bedeutung von Roadshows (relevant für den 1. Call) und Infoveranstaltungen zur Unternehmensansprache hat über die drei Calls hinweg etwas an Bedeutung gewonnen. Dagegen ist die Bedeutung der Wirtschaftskammer zur Ansprache der Unternehmen - speziell im Vergleich zum 1. Call - zurückgegangen. Neu als Informationskanal für den 2. und 3. Call sind die regionalen Netzwerkpartner. 
Grafik 18 Informationskanäle, über die die Einreicher des 2. und 3. Calls von iP erfahren haben, Betriebe in \%

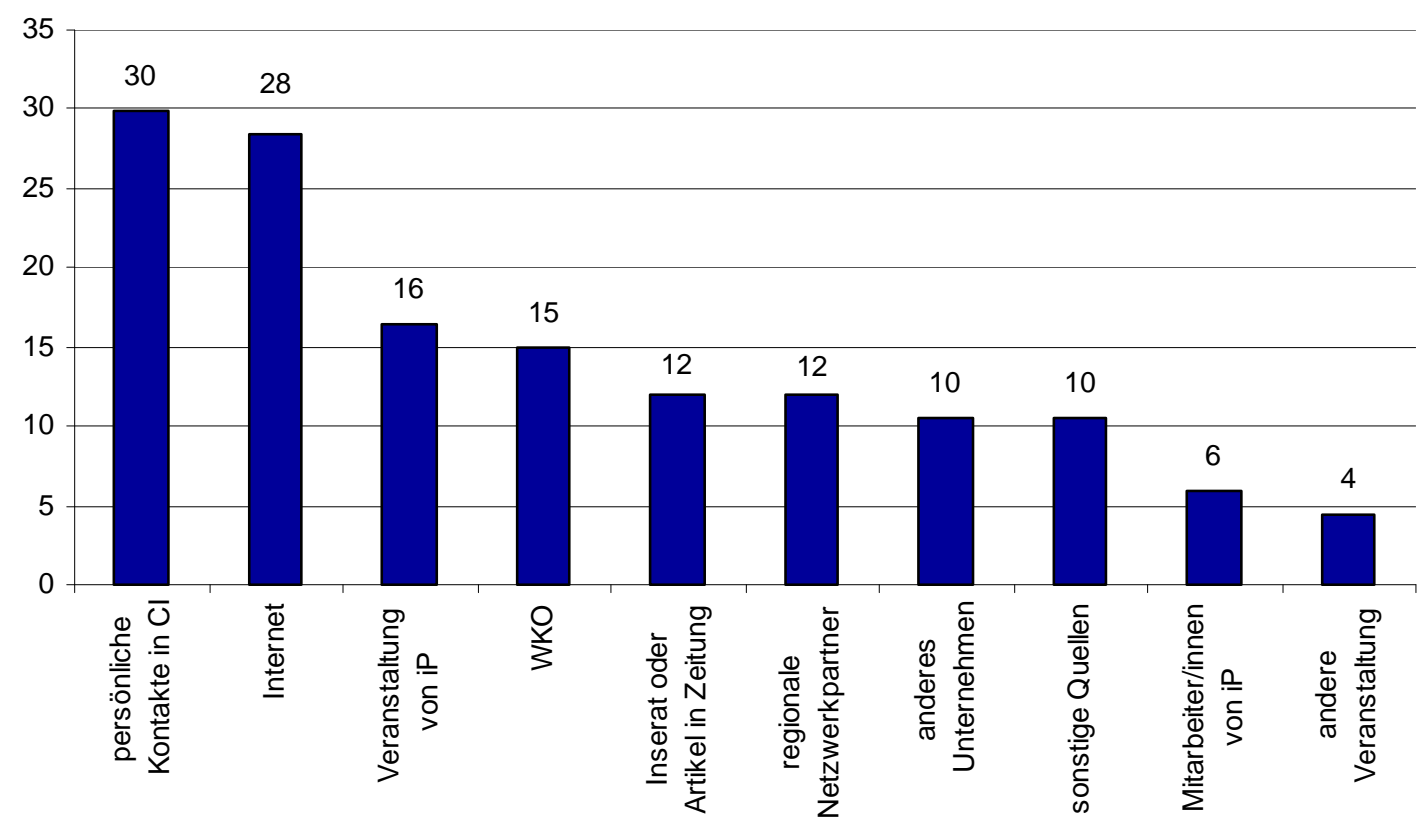

Quelle: KMU FORSCHUNG AUSTRIA, Mehrfachnennungen möglich, $n=67$

Um in Zukunft eine breitere Ansprache der Adressaten des ImpulsProgramm creativwirtschaft zu erwirken, scheint eine Aktivierung der Öffentlichkeitsarbeit, die offensichtlich insbesondere im 1. Call eine deutliche Wirkung gezeigt hat sinnvoll. Eine entsprechende Dotierung und v.a. die Möglichkeit für das Programm auch außerhalb der Kommunikationsschienen der aws, die nur teilweise die Zielgruppe von iP über ihre vorhandenen Informationskanälen erreichen kann, eigene PR-Maßnahmen zu setzen wären in diesem Zusammenhang erste wichtige Schritte. Wie mehrfach angesprochen, sehen sich viele kreativ tätige Personen oder Unternehmer/innen nicht als „klassische“ Wirtschaftstreibende und haben daher vergleichsweise wenige Berührungspunkte zu einer Förderbank wie der aws. In jedem Fall sollte die programmspezifische Homepage von iP (mit eigener Domain) beibehalten und regelmäßig aktualisiert werden. 


\section{Analyse der Programmwirkungen}

In diesem Kapitel werden die Nutzendimension und ersten Outputs der geförderten Projekte im Rahmen des ImpulsProgramm creativwirtschaft auf Projektebene analysiert. Dabei sei darauf hingewiesen, dass bei der gegenständlichen Evaluierung aufgrund der bisherigen Kürze der Laufzeit des Programms zumeist nur die kurzfristig auftretenden Wirkungen erfasst werden können. Von den 26 bezüglich ihrer Wirkungen untersuchten Projekte (untersuchte geförderte Projekte des 1. und 2. Call) befanden sich zum Zeitpunkt der Befragung mehr als die Hälfte noch im Protoypenstadium, und nur 10 Projekte hatten die Produktentwicklung abgeschlossen. Eine Umsetzung der jeweiligen Projektergebnisse in Markterfolge bzw. eine breite Diffusionswirkung der entwickelten Technologien kann daher noch nicht erwartet werden. Ziel der Evaluierung im Bereich der Analyse der Programmwirkungen ist es aus diesem Grund vielmehr, den bisherigen Projektverlauf zu bewerten sowie Anzeichen für längerfristige Auswirkungen hinsichtlich Verhaltensänderungen im Bereich des Aufbaus neuer Kompetenzen zu untersuchen.

\subsection{Nutzen des Programms}

In Hinblick auf den Nutzen des Programms wurde von den befragten Unternehmen vielfach kommuniziert, dass sie iP nicht als Förderung, sondern vielmehr explizit als Wettbewerb sehen. Die „Auszeichnung“ von iP gefördert zu werden bedingt für die Unternehmen den größten Nutzen: Über 80 \% der Unternehmen nannten so auch den Reputationsaufbau als größten Nutzen, den das geförderte Projekt generiert hat (vgl. Grafik 19). Die Unternehmen kommen durch den Erhalt der Förderung vermehrt in die Öffentlichkeit (Veranstaltungen, Homepage, Publikationen, Presseaussendungen von iP). Die „Auszeichnung“ durch iP und der Erhalt finanzieller Mittel für die Verwirklichung eines Projekts sind wichtige Assets, die das Unternehmen zugesprochen bekommt. Dadurch (und vermutlich auch durch die verbundene finanzielle Prüfung für die Förderzusage durch die aws) wird dem Unternehmen z.B. bei Verhandlungen mit Geschäftsbanken geholfen. Dies umso mehr wenn die Marke iP weiter an Bekanntheit gewinnt (siehe Abschnitt 5.3). 
Grafik 19 Nutzendimensionen der iP Förderung bei den geförderten Unternehmen des 1. und 2. Call, Betriebe in \%

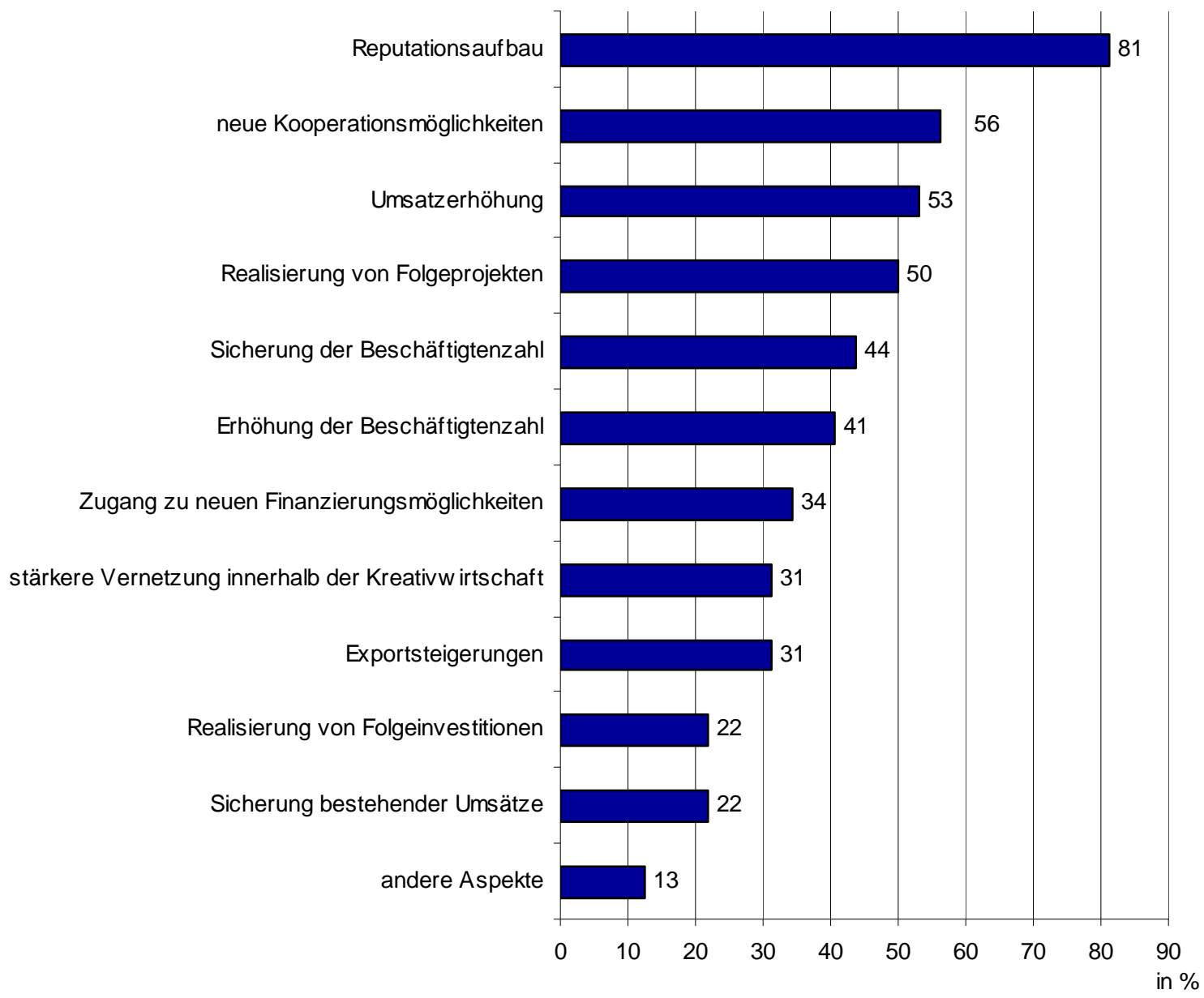

Quelle: KMU FORSCHUNG AUSTRIA, Mehrfachnennungen möglich, $n=32$

Der weitere Nutzen, der durch die Förderung von iP gestiftet wird, unterscheidet sich je nach Bereich aus dem die Unternehmen stammen (wobei eine gewisse Vorsicht bei der Interpretation der Daten anzulegen ist, wegen der tlw. geringen Zahl antwortender Betriebe in den einzelnen Bereichen). Im Musikbereich wurden nach dem Reputationsaufbau als weiterer wichtiger Nutzenaspekt die Vernetzung innerhalb der Kreativwirtschaft, neue Kooperationsmöglichkeiten und Umsatzerhöhung genannt. Im Multimedia-Bereich sind ebenfalls neue Kooperationsmöglichkeiten wichtig sowie die Realisierung von Folgeprojekten. Im Designbereich steht neben dem Reputationsaufbau die Umsatzerhöhung im Mittelpunkt. In diesem Bereich scheint die Vernetzung gar kein Thema zu sein. Insgesamt sind die Nutzendimensionen für die durch iP geförderten Unternehmen sehr vielfältig, neben dem deutlich dominierenden Reputationsaufbau scheint das Programm auch dazu beizutragen, die Kooperations- und Vernetzungskompetenzen der geförderten Unternehmen zu erhöhen und einen wesentlichen Beitrag zur Arbeitsplatz- und Umsatzsicherung bzw. -schaffung in diesen Betreiben zu leisten. Offensichtlich bestehen zudem bereits erste Hinweise auf die Realisierung von Folgeprojekten. Sollte es dem ImpulsProgramm tatsächlich auch gelingen, den Betrieben aus dem Bereich der Kreativwirtschaft den Zugang zu neuen Finanzierungsmöglichkeiten zu erleichtern, wie immerhin von einem guten Drittel der geförderten Unternehmen angegeben, dann wäre das als positiver Hinweis auf die 
Erreichung des Programmziels, nämlich die Steigerung der wirtschaftlichen Kompetenzen in den kreativen Branchen zu werten.

\subsection{Output der geförderten Projekte}

Die geförderten Unternehmen planen auf Basis der von iP geförderten Projekte in erster Linie die Entwicklung von neuen Produkten. Die Entwicklung von Prototypen hierfür ist dabei bei den geförderten Unternehmen am weitesten fortgeschritten, über $50 \%$ der befragten Unternehmen geben dies bereits als Resultat ihrer Entwicklungstätigkeiten an. Neue Produkte wurden bereits von rd. $40 \%$ der im Rahmen des 1. und 2. Calls geförderten Unternehmen entwickelt, weitere $60 \%$ planen dies noch. Die Entwicklung von neuen Verfahren, neuen Dienstleistungen und neuen Vermarktungsstrategien ist von immerhin $65 \%-67 \%$ der im Rahmen von iP geförderten Unternehmen geplant bzw. zum Teil bereit erfolgt (vgl. Grafik 20).

\section{Grafik 20 Resultate der Entwicklungstätigkeiten der geförderten Unternehmen des} 1. und 2. Call, Betriebe in \%

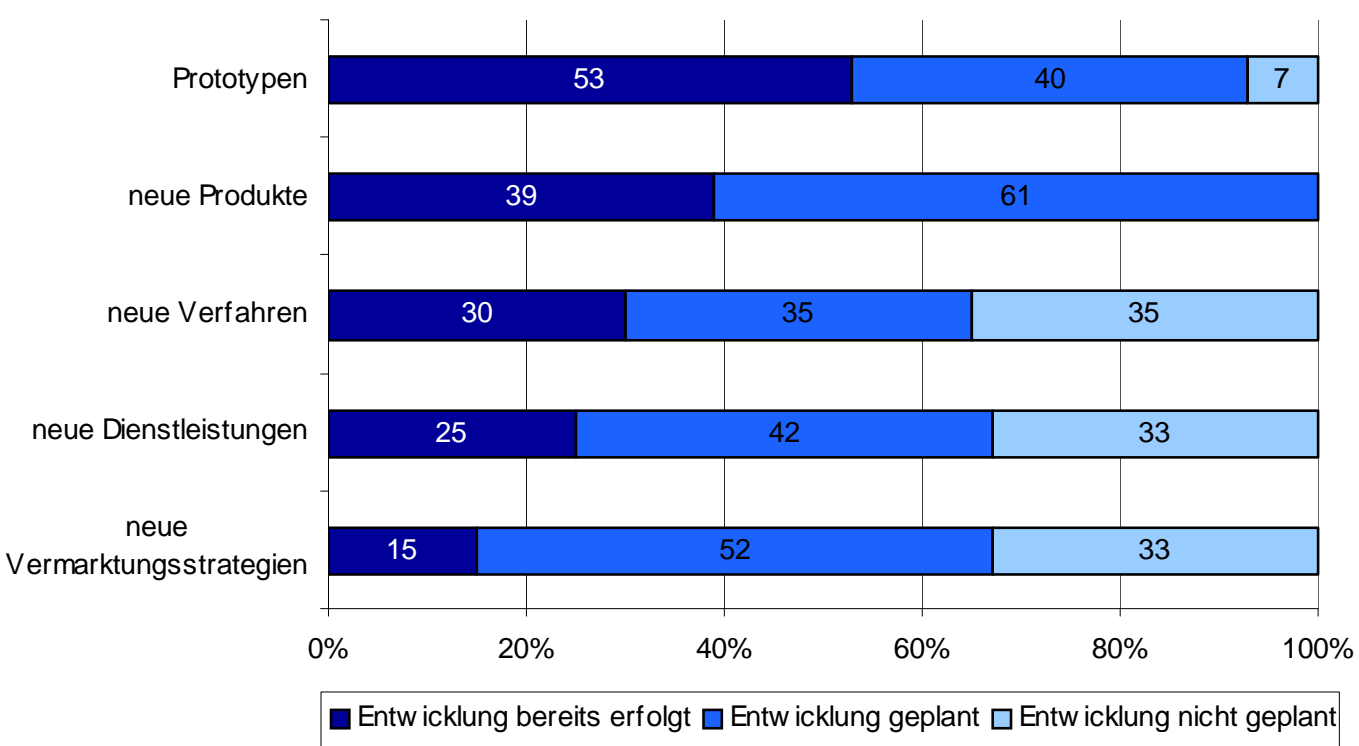

Quelle: KMU FORSCHUNG AUSTRIA, $n=26$

Rd. $40 \%$ der geförderten Unternehmen planen bzw. haben eine Absicherung ihrer Projektergebnisse durch gewerbliche Schutzrechte durchgeführt. Ein Drittel der Unternehmen möchte die Projektergebnisse durch Schutzrechte absichern, weiß jedoch nicht welche Schutzrechtsstrategie dazu am besten geeignet sind. Dieses Ergebnis kann mit dem Bedarf an Weiterbildung in diesem Bereich in Zusammenhang gebracht werden. Weniger als $30 \%$ der geförderten Unternehmen planen keine Eigentumsrechte zu begründen (vgl. Grafik 21). 


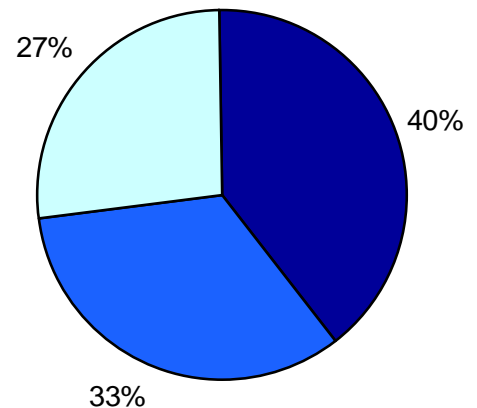

Absicherung durch Schutzrechte geplant oder erfolgt

Absicherung durch Schutzrechte geplant, aber die genaue Ausgestaltung der Schutzrechtsstrategie ist noch nicht entschieden.

$\square$ keine Absicherung durch geistige Egentumsschutzrechte geplant

Quelle: KMU FORSCHUNG AUSTRIA, $n=33$

Die Schutzrechte (unabhängig davon ob eine Anmeldung geplant oder bereits erfolgt ist), von denen am häufigsten Gebrauch gemacht wird, sind Marken und Urheberrechte, gefolgt von Patenten und Gebrauchsmustern. Als letzter Stelle fungiert das registrierte Design (vgl. Tabelle 3).

Tabelle 3 Anzahl begründeter Schutzrechte bei geförderten Projekten des 1. und 2. Call, Absolutzahlen

\begin{tabular}{l|c|c|c|c|c}
\hline & Patente & $\begin{array}{c}\text { Gebrauchs- } \\
\text { muster }\end{array}$ & Marken & $\begin{array}{c}\text { Registriertes } \\
\text { Design }\end{array}$ & $\begin{array}{c}\text { Urheber- } \\
\text { rechte }\end{array}$ \\
\hline Anmeldung geplant & 1 & 1 & 4 & 1 & $\left.3^{*}\right)$ \\
Anmeldung erfolgt & 4 & 4 & 4 & 2 & 3 \\
\hline \hline GESAMT & 5 & 5 & 8 & 3 & 6 \\
\hline
\end{tabular}

*) Copyrights unterliegen für die Entfaltung ihrer Rechtsfolgen normalerweise keiner Anmeldepflicht, wobei aber Ausnahmen bestehen können (z.B. im Musikbereich)

Quelle: KMU FORSCHUNG AUSTRIA

Der bisher realisierte Output der Projekte im Rahmen des Impulsprogramm creativwirtschaft ist als relativ hoch einzustufen, speziell hinsichtlich der Schutzrechte, wenn diese in Relation zu der relativ geringen IPR Nutzungsintensität durch KMU im Allgemeinen, wie sie in zahlreichen Untersuchungen festgestellt wurde, betrachtet werden. Insbesondere der Patentoutput ist bemerkenswert, da Patente bei eher kreativ orientierten Leistungen üblicherweise weniger häufig zum Einsatz kommen (hier wird oft das Technizitätskriterium nicht hinreichend erfültt). Der Patentoutput kann auch als weiteres Indiz gesehen werden, dass es dem ImpulsProgramm creativwirtschaft in hohem Maße gelingt, Projekte mit kreativen Anteilen zu fördern, ohne aber auf einen Technizitätsanspruch zu verzichten. 


\subsection{Additionalität}

Die Additionalität, das heißt, das Ausmaß in dem das entsprechende Projekt von den Unternehmen auch ohne Erhalt der Förderung durchgeführt worden wäre, ist bei iP vergleichsweise hoch. Im 1. Call hätten noch 3 der insgesamt 24 geförderten Unternehmen das Projekt zumindest in überwiegenden Ausmaß auch ohne den Erhalt der Förderung durchgeführt, beim 2. Call hatte die Förderung bei allen befragten Unternehmen die Durchführung des Projekts überhaupt erst ermöglicht (vgl. Grafik 22). Das ImpulsProgramm creativwirtschaft weist somit so gut wie keine Mitnahmeeffekte auf. Ein Tatbestand, der vermutlich auch auf die noch vergleichsweise förderferne Klientel des Programms zurückzuführen ist.

\section{Grafik 22 Additionalität, Anzahl der geförderten Unternehmen absolut}

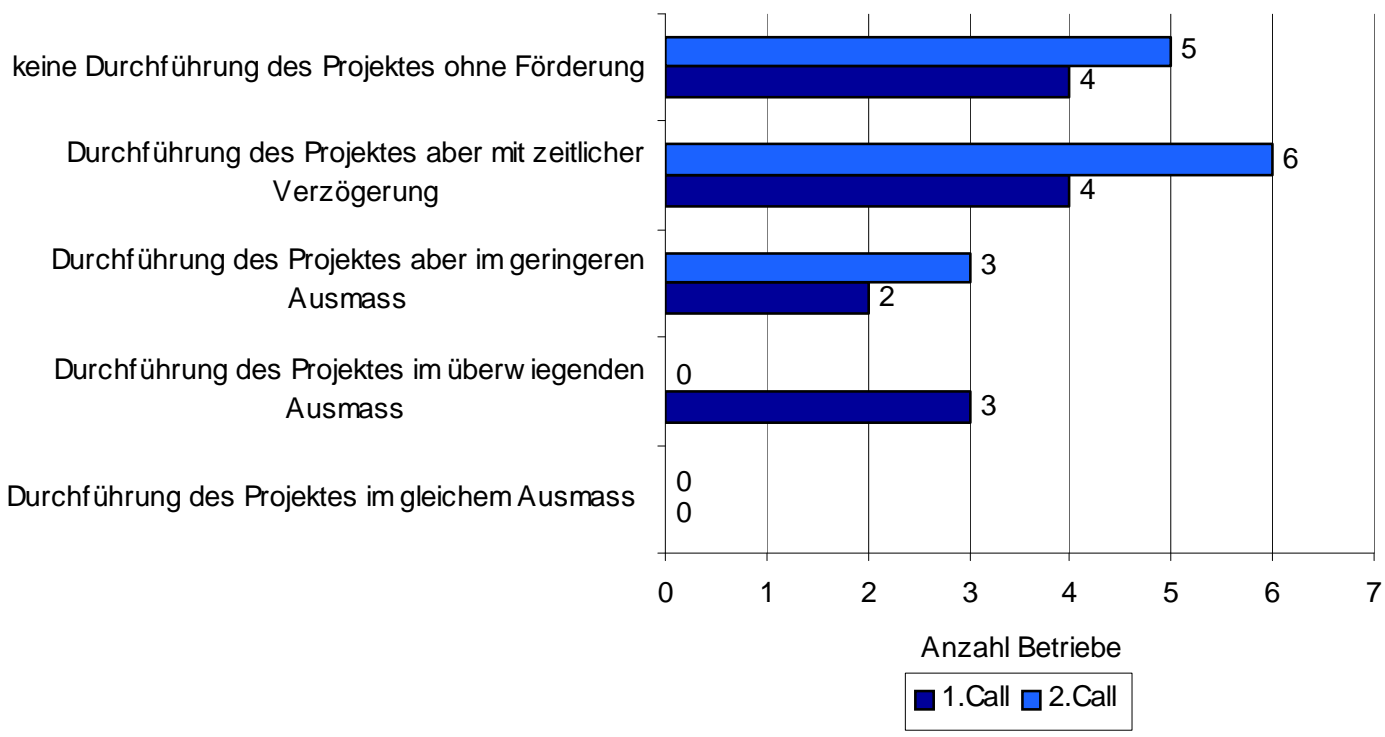

Quelle: KMU FORSCHUNG AUSTRIA, 1.Call: $\mathrm{n}=13$ (aus der ersten Evaluierung), 2.Call=14

Insgesamt wird das Programm von den einreichenden und geförderten Unternehmen gut angenommen. Rd. $70 \%$ der befragten Unternehmen würden nochmals bei iP einreichen. Ein Fünftel der Unternehmen ist sich nicht sicher ob sie nochmals einreichen würden, nur rd. $10 \%$ schließen dies dezidiert aus. 


\section{Zusammenfassung und Schlussfolgerungen}

Im Juni 2007 wurde die KMU FORSCHUNG AUSTRIA von der Austria Wirtschaftsservice $\mathrm{GmbH}$ (aws) beauftragt, eine begleitende Evaluierung des seit 2004 bestehenden ImpulsProgramm creativwirtschaft (iP) durchzuführen. Im Rahmen der entsprechenden Arbeiten war insbesondere zu klären, ob das Förderprogramm hinsichtlich der Erreichung seines Globalzieles - der Steigerung des Innovationspotenzials und der Wettbewerbsfähigkeit von österreichischen KMU in und durch die Bereiche Musik, Multimedia und Design - in seiner Implementierung als effektiv und effizient angesehen werden kann; hinsichtlich etwaiger identifizierter Schwachstellen oder zweckmäßiger Modifikationen/Ausbaumöglichkeiten waren entsprechende Handlungsempfehlungen zu formulieren.

Für die umfassenden Bewertung des Programms wurde auf einen Mix aus quantitativen und qualitativen Methoden zurückgegriffen. Dabei kamen folgende Erhebungsinstrumente zum Einsatz: eine standardisierte Unternehmensbefragung unter 90 Unternehmen, die einen Förderantrag bei iP gestellt haben; 16 offene Interviews mit Programmstakeholdern, Juror/innen und Expert/innen aus den genannten drei Themenbereichen; ein Logic Chart Workshop; sowie Auswertungen vorhandener Programmdokumente. Die vorliegende Zwischenevaluierung ist die zweite für das ImpulsProgramm creativwirtschaft; die Ergebnisse der ersten Zwischenevaluierung aus dem Jahr 2005/2006 wurden als Referenz für einen Vergleich im Zeitverlauf herangezogen. Im Einzelnen ergibt sich aus den Evaluierungsarbeiten (Erhebungszeitraum: Juni 2007 bis Oktober 2007) folgender Befund:

1. Das ImpulsProgramm creativwirtschaft wurde seit der ersten Zwischenevaluierung im Jahr 2005/2006 erheblich weiterentwickelt. Augenscheinlichste Veränderung war die Eingliederung des Programms in das Leistungsportfolio der aws, und die damit verbundene Übersiedlung des Programmbüros vom Museumsquartier im 7. Wiener Gemeindebezirk in die Räumlichkeiten der Austria Wirtschaftsservice (aws). Die aws ist nunmehr alleiniger Träger des Förderprogramms, die arge creativwirtschaft - damals Co-Träger der Initiative - hat seitdem die Rolle eines strategischen Partners inne. Diese organisatorische Änderung ist durchwegs positiv zu beurteilen. Die vollständige Eingliederung in die aws ermöglicht die Nutzung von Synergiepotenzialen zu anderen Förderprogrammen und hilft die Förderabwicklung effektiver und effizienter zu gestalten. Hinsichtlich Zuständigkeiten und Governance ist das Programm klar aufgestellt.

2. Neben der oben beschriebenen organisatorischen Umstellung wurde das Programm auch inhaltlich erweitert. In Ergänzung zur monetären Projektförderung (nunmehr iP Förderung genannt), die über das Instrument des Call abgewickelt wird und immer noch das Herzstück des Programms bildet, wurde speziell das Begleitprogramm ausgebaut: Zum Einen wurde eine von der monetären Förderung unabhängige Workshopschiene ins Leben gerufen, die sich speziell mit betriebswirtschaftlichen Themen der Unternehmensgründung und -führung auseinandersetzt und auf Unternehmen der Creative Industries (Cl) zugeschnitten ist (die sog. „we“-Workshops werden gemeinsam mit der Wiener Förderstelle departure angeboten). Zum Anderen wurden auch Einreichberatungs- und Businessplanworkshops für Betriebe durchgeführt, die einen Antrag auf iP Förderung gestellt und in einer ersten Auswahlrunde positiv bewertet wurden. Weiters tritt die aws im Rahmen von iP als Ko-financier von vier sog. „Leitprojekten“ auf. Dazu zählen Kreatives Handwerk Tirol, Kreatives Handwerk Salzburg, AMAN (Austrian Music Ambassador Network) und das designforum (eine Kompetenz- und Servicestelle für 
den Designbereich im Wiener Museumsquartier). Diese „Leitprojekte“ können selbst als regional- und/oder $\mathrm{Cl}$-themenspezifische Förderprogramme angesehen werden. Schließlich sind auch Änderungen in Bezug auf Abwicklungsstrukturen (hier vor allem die Schaffung eines Expert/innenbeirats) sowie die Adaption der Richtlinien und Flyer- bzw. Homepagetexte zu nennen, die mittels der gewonnen Erfahrungen und Ergebnisse der ersten Zwischenevaluierung angepasst wurden.

3. Die iP Förderung bildet das Kernelement des ImpulsProgramm creativwirtschaft. Im Rahmen der iP Förderung werden Projekte, die kreativen und innovativen Charakter aufweisen und marktorientiert sind, sprich bei der Umsetzung eine erfolgreiche wirtschaftliche Verwertung erwarten lassen, mit bis zu 70 \% der förderbaren Kosten unterstützt. Die Projektvergabe erfolgte bislang im Rahmen von Calls, von denen bis dato drei durchgeführt wurden. Im Zeitraum 2004/2005 fand der erste Call mit insgesamt 315 eingereichten Anträgen und 24 geförderten Projekten (Fördersumme gesamt: rd. € 2,1 Mio) statt; der zweite im Zeitraum 2005/2006 mit insgesamt 159 Anträgen und 16 geförderten Projekten (ausgeschüttete Fördermittel: rd. $€ 1,3$ Mio); der dritte Call fand 2007 statt und wies 123 Einreichungen auf. In Summe stammen die meisten Anträge aus Wien, gefolgt von, mit deutlichem Abstand, der Steiermark und Salzburg. Der zu beobachtende Rückgang der eingereichten Anträge ist zum Einen vermutlich auf Lerneffekte in der Cl Community zurückzuführen (die wiederum auch von der Präzisierung des Fördertatbestandes in den Programmrichtlinien herrühren) und, speziell auf den 1. Call bezogen, zum Anderen auf einen Projekt- und Ideenstau der sich vor Initiierung des Förderprogramms, in Ermangelung alternativer Förderschienen, gebildet haben dürfte.

4. In diesem Zusammenhang wurde von Expert/innen immer wieder festgestellt, dass zahlreiche gute Ideen in der $\mathrm{Cl}$ Community existieren die aber noch nicht so weit gediehen sind als dass Sie den Ausleseprozess bei einem iP Call positiv durchlaufen könnten. Hier wäre die Etablierung eines Segments im ImpulsProgramm creativwirtschaft anzudenken (eine mögliche „iP Light“-Schiene) um derartige Protoprojekte anzuschieben. Bei einer derartigen „iP Light“ Förderung muss aber auch die Herausforderung berücksichtigt werden, den Threshold für die Inanspruchnahme der Förderung nicht so niedrig zu gestalten, dass die Zahl der dann einlangenden Anträge durch zu hohe Kosten bzw. zu hohen administrativen Aufwand für die Antragsbearbeitung den Nutzen der Programmschiene konterkariert.

5. Neben der zahlenmäßigen Entwicklung der Anträge im Rahmen der drei Calls zeigen sich im Zeitverlauf auch Änderungen (i) hinsichtlich der Struktur der antragstellenden Betriebe sowie (ii) in Bezug auf die Qualität der Anträge. Letztere dürfte, den interviewten Juror/innen zu Folge, deutlich gestiegen sein. Bezogen auf die Zusammensetzung der an iP teilnehmenden Betriebe ist festzustellen, dass im ersten Call $28 \%$ der antragstellenden Unternehmen angaben, anlässlich der Teilnahme an iP gegründet worden zu sein. Im 2. und 3. Call sank der Anteil derartiger Anlassgründungen auf im Durchschnitt etwa $9 \%$ - damit setzt sich das ImpulsProgramm creativwirtschaft nun deutlicher als zuvor von Gründungsförderungsprogrammen ab und fokussiert, im Sinne eines Streamlinings der Programmziele, stärker auf bereits etablierte Unternehmen. Darüber hinaus ist festzustellen, dass die einreichenden Betriebe der Struktur der Creative Industries entsprechend überwiegend kleinbetrieblich strukturiert ( $87 \%$ hatten maximal 10 Beschäftigte) und sehr jung sind: Die Hälfte der antragstellenden Unternehmen wurde nach 2003 gegründet. 
6. Ein wesentliches Ergebnis der ersten Zwischenevaluierung war, dass fast die Hälfte der Betriebe (48\%) vor iP bereits Förderungen in Anspruch genommen hatte; dieser Anteil ist im 2. Call auf $27 \%$ gesunken, betrug aber im 3. Call wieder $55 \%$. Zudem hat sich der Anteil von Unternehmen, die Preise gewonnen haben, wie sie in kreativ-künstlerischen Bereichen vergeben werden, von $3 \%$ im 1 . Call auf $21 \%$ im 3. Call erhöht. Dies könnte als Indikator für die zuvor genannte gestiegene Qualität der Anträge gedeutet werden.

7. Im Rahmen der Evaluierung war auch der Frage nach der Bekanntheit von iP in der $\mathrm{Cl}$ Community und deren Entwicklung im zeitlichen Verlauf nachzugehen. Diese Frage konnte nur indirekt, über die subjektive Einschätzung der befragten Unternehmen und Expert/innen, beantwortet werden. Es ergibt sich das Bild, dass knapp die Hälfte der befragten Personen das Programm als „sehr bekannt“ oder „eher bekannt“ einschätzen; generell gehen die meisten Interviewpartner davon aus, dass die Bekanntheit von iP in den vergangenen Jahren leicht gestiegen ist, aber noch durchaus Potenzial für eine weitere Diffundierung der Marke „iP“ in der Zielgruppe besteht.

8. Als wichtigste „Vertriebskanäle“ für das ImpulsProgramm creativwirtschaft können das Internet (die ImpulsProgramm-Homepage) und persönliche Kontakte in der $\mathrm{Cl}$ Community angesehen werden; vor diesem Hintergrund zeigt sich die Wichtigkeit der pro-aktiven Ansprache der $\mathrm{Cl}$ Community, wie etwa durch die Beibehaltung einer programmspezifischen Homepage. Letztlich ergibt sich die Notwendigkeit einer gezielten Ansprache auch dadurch, dass sich viele kreativ tätige Personen oder Unternehmer/innen nicht als „klassische“ Wirtschaftstreibende betrachten und daher vergleichsweise wenige Berührungspunkte zu einer Förderbank wie der aws haben.

9. Das in der ersten begleitenden Evaluierung erwähnte Spannungsfeld zwischen technisch orientierter Innovationsförderung einerseits und Kreativförderung andererseits war im 2. und 3. Call so nicht mehr zu beobachten. Augenscheinlich ist es gelungen, die Richtlinientexte derart umzuformulieren und die Änderungen auch in entsprechender Weise zu kommunizieren, dass sowohl unter den Juror/innen und Expert/innen als auch bei den antragstellenden Unternehmer/innen weitestgehende Klarheit über die förderbaren Tatbestände/Projektinhalte besteht. Aus inhaltlicher Sicht ist positiv zu unterstreichen, dass der Kreativitätsaspekt zwar weiter betont wurde, ohne aber auf das produktbezogene Technizitäts- und Innovationskriterium völlig zu verzichten. Insgesamt weist die Gruppe der iP nutzenden Betriebe einen begrüßenswerten Mix an Unternehmensaktivitäten auf: Neben Betrieben, die sich selbst als Unternehmen sehen, die hauptsächlich „künstlerisch-kreative“ Produkte oder Dienstleistungen anbieten, werden dabei auch Betriebe gefördert, die sich als Zulieferunternehmen für Kreative begreifen. Weiters finden sich auch Unternehmen, die eher traditionellen Branchen zuzuordnen sind, aber sich z.B. über eine kreative Designleistung neue Fähigkeiten aneignen wollen, einerseits um eine Weiterentwicklung inres Betriebs zu initiieren, andererseits um Wettbewerbsvorteile zu lukrieren. 
10. Der größte Nutzen des Programms liegt für die Betriebe vor allem im Reputationsaufbau. Das ImpulsProgramm creativwirtschaft wird von vielen Betrieben explizit als Wettbewerb wahrgenommen und der Erhalt der Förderung als „Auszeichnung“ angesehen, welche im Rahmen von Eigenmarketing bei Verhandlungen mit Kund/innen und Kapitalgebern auch genutzt wird. Diese positive Wahrnehmung der Marke „iP“ ist auch Ergebnis des selektiven Auswahlprozesses im zweiphasigen Callverfahren, welches aber auch gewisse Nachteile aufweist: Die Zeitdauer bis ein Antrag bewilligt ist, wird vielfach als zu lang empfunden, speziell vor dem Hintergrund, dass in den Creative Industries Produktlebenszyklen sehr kurz sein können und "time to market" ein wesentlicher Erfolgsfaktor darstellt. Vor diesem Hintergrund ist die diskutierte Umstellung auf ein einphasiges Callsystem (d.h. ein Auswahlverfahren mit nur einer Auswahlrunde anstelle von derzeit zwei), das häufigere Einreichtermine vorsieht sinnvoll, wenn gleichzeitig sichergestellt wird, dass es bei den Projektauswahl- und -bewertungsverfahren nicht zu Qualitätsverlusten kommt.

11. Die Zufriedenheit der Einreicher/innen mit unterschiedlichen Aspekten der Programmabwicklung, wie beispielsweise die Erreichbarkeit der iP Mitarbeiter/innen, die Qualität der Informationsunterlagen oder der abgehaltenen Informationsveranstaltungen ist sehr hoch. Allgemein wird das Engagement der iP Mitarbeiter/innen deutlich hervorgehoben, insbesondere was die gemeinsam mit $\mathrm{Cl}$ Expert/innen erfolgte Erarbeitung von Konzepten für die operative und strategische Weiterentwicklung des Förderprogramms betrifft. Differenzierter zeigen sich die Antworten hinsichtlich der Zufriedenheit mit den verschiedenen Aspekten der Calldurchführung. Werden die Klarheit der Informationsunterlagen für die einzelnen Calls (Formulare), die Zeit, die für die Antragstellung zur Verfügung stand oder die Betreuung während der Antragsstellung durch das iP Team noch als zufriedenstellend beurteilt, so fällt die Bewertung der Bearbeitungsdauer sowie des administrativen Aufwands bei der Antragserstellung deutlich schlechter aus. Während der Bereich des administrativen Aufwands vorsichtig zu bewerten ist - ein bestimmtes $\mathrm{Maß}$ an administrativen Aufwand ist meist unumgänglich - muss die häufig bemängelte lange Bearbeitungsdauer vor dem Hintergrund, dass „time to market“ in vielen $\mathrm{Cl}$ Bereichen ein essentieller Erfolgsfaktor ist, kritischer gesehen werden.

12. Wie in der ersten Zwischenevaluierung gab es besonders schlechte Noten für die „Transparenz der Beurteilungskriterien“ und für das „Feedback bei Ablehnung der Anträge“. Da bei der Beurteilung von Kreativitätsleistungen immer ein gewisser subjektiver Anteil mitentscheidend ist, werden diese miteinander verbundene Themenkomplexe eine Herausforderung für $\mathrm{Cl}$ Förderprogramme bleiben - gerade deswegen jedoch sollten deutliche Anstrengungen unternommen werden, Entscheidungen und deren Grundlagen genau darzulegen (diskussionsfähig wären gem. Expert/innen in dieser Hinsicht z.B. kostengünstige Medien wie Internetforen oder Blogs, die dazu benutzt werden könnten zumindest Eindrücke zum Unterschied zwischen erfolgreichen und nicht erfolgreichen Anträgen zu kommunizieren und zu diskutieren), damit letztlich die Akzeptanz bei der Zielgruppe in breitem Maße erhalten bleibt.

13. Bezüglich der Begleitmaßnahmen ist festzustellen, dass diese zwar erheblich weiterentwickelt wurden, der Zuspruch der Businessplan-, Einreichberatungs- und vor allem der „we“- Workshops jedoch noch unter den Erwartungen der Programmverantwortlichen liegt. Der Hauptgrund, den die Unternehmen für die Nicht-Nutzung der bzw. die Nicht-Teilnahme an den Begleitmaßnahmen angaben, war, dass sie bereits über die zu vermittelnden Kenntnisse verfügen. Jene Unternehmen, die hingegen Workshops besucht haben, waren mit diesen durchwegs zufrieden. 
Expert/innen zufolge ist die positive Selbsteinschätzung der Unternehmer/innen betreffend ihrer Vorkenntnisse hinsichtlich der Workshopthemen in vielen Fällen durchaus hinterfragenswert - daraus ließe sich, als Herausforderung, ein gewisser Sensibilisierungsbedarf ableiten.

14. Vorgebrachte Verbesserungsvorschläge der Unternehmen wie auch der Expert/innen betreffend Begleitmaßnahmen behandelten vor allem fehlende Angebote in Bezug auf Beratung im Bereich der gewerblichen Schutzrechte für geistiges Eigentum, Internationalisierungs- und Vermarktungsförderung (hier wurde oftmals vorgebracht, dass $\mathrm{Cl}$ Produkte und Dienstleistungen sich meist in einem globalen Wettbewerb befinden und Hilfe für das Auftreten auf globaler Ebene wichtig ist) sowie Vernetzung/Kooperation. Mit Ausnahme des Themenfeldes „Schutz geistigen Eigentums" sind jedoch die angesprochenen Themen nur unzureichend mit dem derzeitigen Instrumentarium von iP abzudecken. Hier gilt es, die sehr breiten Wünsche der Unternehmer/innen selektiv hinsichtlich deren Zweckmäßigkeit für iP zu prüfen; viele Themenstellungen können, vor dem Hintergrund von Budgetrestriktionen, sicher nicht ohne erhebliche Ressourcenausweitung vernünftig angesprochen werden. Bei manchen Themenstellungen (z.B. hinsichtlich Vermarktung) sind zudem beihilfenrechtliche Bestimmungen zu beachten.

15. Die gute Performance des Programms lässt sich auch an der hohen Additionalität bzw. dem Fehlen reiner Mitnahmeeffekte ablesen: Bei einigen Projekten wäre zwar mit zeitlichen Verzögerungen oder geringeren Projektumfängen bei fehlender Förderung zu rechnen gewesen, aber kein Projekt wäre ohne Förderung in zum Antrag äquivalenter Weise vollinhaltlich durchgeführt worden. Dies ist als Zeichen für die hohe Relevanz des Programms zu werten. Ebenso positiv: Etwa $70 \%$ der Einreicher/innen gaben an, dass sie eine nochmalige Einreichung bei iP in Erwägung ziehen würden; nur $11 \%$ schlossen dies dezidiert aus. 


\section{Anhang}

\section{Liste der Interviewpartner/innen}

\begin{tabular}{|c|c|c|c|}
\hline $\mathbf{N r}$ & Name & Institution & Funktion \\
\hline \multicolumn{4}{|c|}{ aws / iP } \\
\hline 1 & $\begin{array}{c}\text { Sonja } \\
\text { Hammerschmid }\end{array}$ & aws / iP & $\begin{array}{c}\text { Bereichsleiterin Innovation \& } \\
\text { Technologie }\end{array}$ \\
\hline 2 & Sabine Pümpel & aws / iP & Projektleitung \\
\hline 3 & Kurt Leutgeb & aws & $\begin{array}{c}\text { Bereichsleiter/Prokurist Förderung \& } \\
\text { Finanzierung }\end{array}$ \\
\hline 4 & Karl Schiller & aws & $\begin{array}{c}\text { Gründungsservice, Betreuung } \\
\text { "plan4you"-Tool, iP Businessplan } \\
\text { Workshop }\end{array}$ \\
\hline \multicolumn{4}{|c|}{ iP Fachjury } \\
\hline 5 & Alexander Grünsteidl & Digital wellbeing labs & Geschäftsführer \\
\hline 6 & Nada Nasrallah & Soda Design & Geschäftsführerin \\
\hline 7 & Glen Oliver Löw & $\begin{array}{l}\text { Industrial Design / } \\
\text { Hochschule für } \\
\text { bildende Künste } \\
\text { Hamburg }\end{array}$ & $\begin{array}{l}\text { Geschäftsführer / Professor für } \\
\text { Produktentwicklung und -gestaltung }\end{array}$ \\
\hline 8 & Horst Hörtner & AEC Future Lab & Geschäftsführer \\
\hline 9 & Rudi Ortner & FM4 & Musikproduzent \\
\hline 10 & Tim Renner & $\begin{array}{l}\text { Motor Entertainment } \\
\text { GmbH \& Co KG }\end{array}$ & Geschäftsführer \\
\hline 11 & Paul Woog & $\begin{array}{l}\text { Popbüro Region } \\
\text { Stuttgart }\end{array}$ & Leiter \\
\hline \multicolumn{4}{|c|}{ iP Beirat } \\
\hline 12 & Gertraud Leimüller & $\begin{array}{c}\text { winnovation } \\
\text { consulting GmbH / } \\
\text { arge creativwirtschaft } \\
\text { austria }\end{array}$ & $\begin{array}{c}\text { Geschäftsführerin / Vorsitzende arge } \\
\text { creativwirtschaft austria }\end{array}$ \\
\hline 13 & Stephan Dorfmeister & $\begin{array}{c}\text { Dorfmeister } \\
\text { Projektentwicklung } \\
\text { GmbH/ arge } \\
\text { creativwirtschaft } \\
\text { austria }\end{array}$ & $\begin{array}{l}\text { Geschäftsführer / stv. Vorsitzender } \\
\text { arge creativwirtschaft austria }\end{array}$ \\
\hline 14 & Andy Pongratz & $\begin{array}{l}\text { X-Art ProDivision } \\
\text { GmbH }\end{array}$ & Geschäftsführer \\
\hline 15 & Georg Tomandl & $\begin{array}{l}\text { Tomandl \& Janisch } \\
\text { OEG }\end{array}$ & Geschäftsführer \\
\hline \multicolumn{4}{|c|}{ Netzwerkpartner } \\
\hline 16 & Christian Mathes & CAST Tyrol & Geschäftsführer \\
\hline
\end{tabular}

